\title{
Staging Parkinson's Disease Combining Motor and Nonmotor Symptoms Correlates with Disability and Quality of Life
}

\author{
D. Santos García (D), ${ }^{1}$ T. De Deus Fonticoba, ${ }^{2}$ J. M. Paz González, ${ }^{1}$ C. Cores Bartolomé, ${ }^{1}$ \\ L. Valdés Aymerich, ${ }^{1}$ J. G. Muñoz Enríquez, ${ }^{1}$ E. Suárez, ${ }^{2}$ S. Jesús, ${ }^{3,4}$ M. Aguilar, ${ }^{5}$ P. Pastor, ${ }^{5}$ \\ L. L. Planellas, ${ }^{6}$ M. Cosgaya, ${ }^{6}$ J. García Caldentey, ${ }^{7}$ N. Caballol, ${ }^{8}$ I. Legarda, ${ }^{9}$ \\ J. Hernández Vara, ${ }^{10}$ I. Cabo, ${ }^{11}$ L. López Manzanares, ${ }^{12}$ I. González Aramburu, ${ }^{4,13}$ \\ M. A. Ávila Rivera, ${ }^{14}$ M. J. Catalán, ${ }^{15}$ V. Nogueira, ${ }^{16}$ V. Puente, ${ }^{17}$ J. M. García Moreno, ${ }^{18}$ \\ C. Borrué, ${ }^{19}$ B. Solano Vila, ${ }^{20}$ M. Álvarez Sauco, ${ }^{21}$ L. Vela, ${ }^{22}$ S. Escalante, ${ }^{23}$ E. Cubo, ${ }^{24}$ \\ F. Carrillo Padilla, ${ }^{25}$ J. C. Martínez Castrillo, ${ }^{26}$ P. Sánchez Alonso, ${ }^{27}$ M. G. Alonso Losada, ${ }^{28}$ \\ N. López Ariztegui $\mathbb{D}^{29}{ }^{29}$ I. Gastón, ${ }^{30}$ J. Kulisevsky, ${ }^{4,31}$ M. Blázquez Estrada, ${ }^{32}$ M. Seijo, ${ }^{11}$ \\ J. Rúiz Martínez, ${ }^{33}$ C. Valero, ${ }^{34}$ M. Kurtis, ${ }^{35}$ O. de Fábregues, ${ }^{10}$ J. González Ardura, ${ }^{36}$ \\ C. Ordás, ${ }^{37}$ L. López Díaz, ${ }^{38}$ P. Mir, ${ }^{4,3}$ P. Martinez-Martin $\left(\mathbb{D},{ }^{4}\right.$ \\ and COPPADIS Study Group ${ }^{39}$
}

${ }^{1}$ CHUAC, Complejo Hospitalario Universitario de A Coruña, A Coruña, Spain

${ }^{2}$ CHUF, Complejo Hospitalario Universitario de Ferrol, A Coruña, Spain

${ }^{3}$ Unidad de Trastornos del Movimiento, Servicio de Neurología y Neurofisiología Clínica, Instituto de Biomedicina de Sevilla, Hospital Universitario Virgen del Rocío/CSIC/Universidad de Sevilla, Seville, Spain

${ }^{4}$ CIBERNED (Centro de Investigación Biomédica en Red Enfermedades Neurodegenerativas), Madrid, Spain

${ }^{5}$ Hospital Universitari Mutua de Terrassa, Terrassa, Spain

${ }^{6}$ Hospital Clínic de Barcelona, Barcelona, Spain

${ }^{7}$ Centro Neurológico Oms 42, Palma de Mallorca, Spain

${ }^{8}$ Consorci Sanitari Integral, Hospital Moisés Broggi, Sant Joan Despí, Barcelona, Spain

${ }^{9}$ Hospital Universitario Son Espases, Palma de Mallorca, Spain

${ }^{10}$ Hospital Universitario Vall d'Hebron, Barcelona, Spain

${ }^{11}$ Complejo Hospitalario Universitario de Pontevedra (CHOP), Pontevedra, Spain

${ }^{12}$ Hospital Universitario La Princesa, Madrid, Spain

${ }^{13}$ Hospital Universitario Marqués de Valdecilla, Santander, Spain

${ }^{14}$ Consorci Sanitari Integral, Hospital General de L'Hospitalet, L'Hospitalet de Llobregat, Barcelona, Spain

${ }^{15}$ Hospital Universitario Clínico San Carlos, Madrid, Spain

${ }^{16}$ Hospital Da Costa, Burela, Lugo, Spain

${ }^{17}$ Hospital del Mar, Barcelona, Spain

${ }^{18}$ Hospital Universitario Virgen Macarena, Sevilla, Spain

${ }^{19}$ Hospital Infanta Sofía, Madrid, Spain

${ }^{20}$ Institut d'Assistència Sanitària (IAS) - Institut Català de la Salut, Girona, Spain

${ }^{21}$ Hospital General Universitario de Elche, Elche, Spain

${ }^{22}$ Fundación Hospital de Alcorcón, Madrid, Spain

${ }^{23}$ Hospital de Tortosa Verge de la Cinta (HTVC), Tortosa, Tarragona, Spain

${ }^{24}$ Complejo Asistencial Universitario de Burgos, Burgos, Spain

${ }^{25}$ Hospital Universitario de Canarias, San Cristóbal de la Laguna, Santa Cruz de Tenerife, Spain

${ }^{26}$ Hospital Universitario Ramón y Cajal, Madrid, Spain

${ }^{27}$ Hospital Universitario Puerta de Hierro, Madrid, Spain

${ }^{28}$ Hospital Álvaro Cunqueiro, Complejo Hospitalario Universitario de Vigo (CHUVI), Vigo, Spain

${ }^{29}$ Complejo Hospitalario de Toledo, Toledo, Spain

${ }^{30}$ Complejo Hospitalario de Navarra, Pamplona, Spain

${ }^{31}$ Hospital de Sant Pau, Barcelona, Spain 


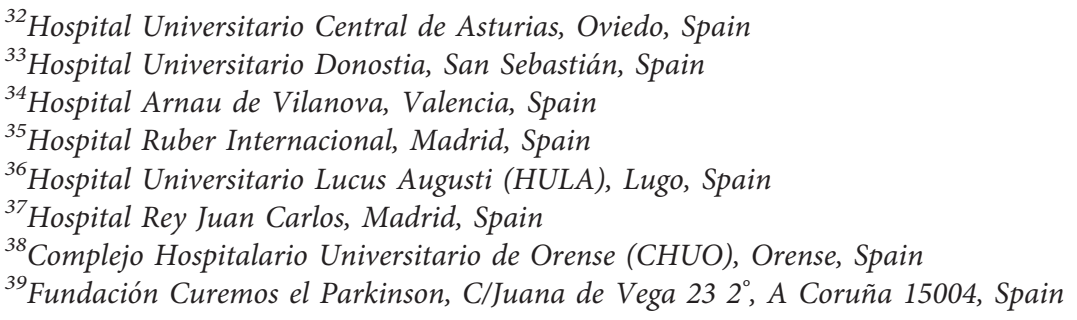

Correspondence should be addressed to D. Santos García; diegosangar@yahoo.es

Received 13 September 2020; Revised 4 November 2020; Accepted 21 February 2021; Published 15 May 2021

Academic Editor: Graziella Madeo

Copyright (c) 2021 D. Santos García et al. This is an open access article distributed under the Creative Commons Attribution License, which permits unrestricted use, distribution, and reproduction in any medium, provided the original work is properly cited.

Introduction. In a degenerative disorder such as Parkinson's disease (PD), it is important to establish clinical stages that allow to know the course of the disease. Our aim was to analyze whether a scale combining Hoehn and Yahr's motor stage (H\&Y) and the nonmotor symptoms burden (NMSB) (assessed by the nonmotor symptoms scale (NMSS)) provides information about the disability and the patient's quality of life (QoL) with regard to a defined clinical stage. Materials and Methods. Cross-sectional study in which 603 PD patients from the COPPADIS cohort were classified according to H\&Y (1, stage I; 2, stage II; 3, stage III; 4, stage IV/V) and NMSB (A: NMSS = 0-20; B: NMSS = 21-40; C: NMSS = 41-70; D: NMSS $\geq 71$ ) in 16 stages (HY.NMSB, from 1A to 4D). QoL was assessed with the PDQ-39SI, PQ-10, and EUROHIS-QOL8 and disability with the Schwab\&England ADL (Activities of Daily Living) scale. Results. A worse QoL and greater disability were observed at a higher stage of H\&Y and NMSB $(p<0.0001)$. Combining both (HY.NMSB), patients in stages $1 \mathrm{C}$ and $1 \mathrm{D}$ and $2 \mathrm{C}$ and $2 \mathrm{D}$ had significantly worse QoL and/or less autonomy for $\mathrm{ADL}$ than those in stages $2 \mathrm{~A}$ and $2 \mathrm{~B}$ and $3 \mathrm{~A}$ and $3 \mathrm{~B}$, respectively $(p<0.005$; e.g., PDQ-39SI in $1 \mathrm{D}$ [ $n=15]$ vs $2 \mathrm{~A}$ [ $n=101$ ]: $28.6 \pm 17.1$ vs $7.9 \pm 5.8 ; p<0.0001)$. Conclusion. The HY.NMSB scale is simple and reflects the degree of patient involvement more accurately than the H\&Y. Patients with a lower H\&Y stage may be more affected if they have a greater NMS burden.

\section{Introduction}

Parkinson's disease (PD) is a progressive neurodegenerative disorder causing motor and nonmotor symptoms (NMS) that result in disability, loss of patient autonomy, and caregiver burden [1]. In a degenerative disease, it is important to establish clinical stages that allow the determination of disease progression for a patient based on different specific symptoms. Ideally, this clinical graduation should be simple to carry out so that it can be used universally in clinical practice. In the case of PD, and based on the classic motor symptoms of the disease, the Hoehn and Yahr (H\&Y) scale is used to describe the progression of PD [2]. The scale was originally described in 1967 and included stages 1 through 5 . It has since been modified with the addition of stages 1.5 and 2.5 to help describe the intermediate course of the disease [3]. This rating system has been largely supplemented by, firstly, the Unified Parkinson's Disease Rating Scale (UPDRS) [4], and more recently, the MDS-Unified Parkinson's Disease Rating Scale (MDS-UPDRS) [5], which assess limitation of Activities of Daily Living (ADL) and NMS. However, evaluating the patient using the UPDRS and/or MDS-UPDRS takes time; specialization is required and, importantly, do not allow the patient to be classified into a clearly differentiated stage, and several NMS are not included. Validated tools for assessing NMS such as the NMSQuest [6] and the nonmotor symptoms scale (NMSS) [7] are used both in trials and in clinical practice. Furthermore, it has been demonstrated that NMS are an important determinant and deteriorating factor of the quality of life (QoL) of PD patients $[8,9]$. Not only motor symptoms but also NMS increase in their severity and burden over time, increasing patients' disability, with additional worsening of their QoL, as well as caregivers' burden and consequential consumption of social resources by increasing societal costs. That is why for staging PD it would be necessary to combine a motor with a nonmotor scale, which would allow the patient to be classified into stages considering both the degree of motor and nonmotor involvement.

Recently, it has been suggested that gradation of PD according to the motor impairment and burden of NMS is an unmet need for an appropriate management of patients [10]. Ray Chaudhuri et al. proposed a PD classification by H\&Y staging and NMS burden level and demonstrated a correlation of both H\&Y staging and NMS burden to disability and QoL [11]. However, QoL and autonomy for ADL regarding the stage considering both together, motor and nonmotor stages, were not analyzed. The H\&Y scale provides quick information about the patient's condition, but since it does not include NMS, it is not very sensitive to reflect the real impact of that condition. Our hypothesis is 
that a patient with a lower H\&Y stage but a greater NMS burden may present a worse QoL and greater disability than another patient with a more advanced $H \& Y$ stage but a lower NMS burden, so it would be beneficial to combine both aspects on a scale. The aim of this study was to classify PD patients from the COPPADIS cohort [12, 13], regarding $\mathrm{H} \& \mathrm{Y}$ and NMS burden combined in a specific scale (HY.NMSB), and to compare QoL and autonomy for ADL between patients in a different HY.NMSB stages.

\section{Materials and Methods}

PD patients recruited from 35 centers of Spain from the COPPADIS cohort [13] from January 2016 to November 2017 were included in the study. Methodology about COPPADIS2015 study can be consulted in https://bmcneurol. biomedcentral.com/articles/10.1186/s12883-016-0548-9.

This is a multicenter, observational, longitudinal-prospective, 5-year follow-up study designed to analyze disease progression in a Spanish population of PD patients. The data for the present study (cross-sectional study) were obtained from the baseline evaluation. All patients included were diagnosed according to UK PD Brain Bank criteria. Exclusion criteria were as follows: non-PD parkinsonism, dementia (Mini Mental State Examination (MMSE) <26), age $<18$ or $>75$ years, inability to read or understand the questionnaires, to be receiving any advanced therapy (continuous infusion of levodopa or apomorphine and/or with deep brain stimulation), and the presence of comorbidity, sequelae, or any disorder that could interfere with the assessment.

Information on sociodemographic aspects, factors related to $\mathrm{PD}$, comorbidity, and treatment was collected. Motor and NMS were evaluated using different validated scales [12]. In patients with motor fluctuations, the motor assessment (H\&Y and UPDRS) was conducted during the OFF state (without medication in the last 12 hours; H\&YOFF and UPDRS-III-OFF) and during the ON state (H\&Y$\mathrm{ON}$ and UPDRS-III-ON). However, in patients without motor fluctuations, it was only performed without medication (first thing in the morning without taking medication in the previous 12 hours). Moreover, in PD patients with motor fluctuations, the nonmotor assessment was conducted during the ON state [12]. The NMSS [7] was used for assessing NMS. This includes 30 items, each with a different nonmotor symptom. The symptoms refer to the 4 weeks prior to assessment. The total score for each item is the result of multiplying the frequency ( 0 , never; 1 , rarely; 2 , often; 3 , frequent; 4 , very often) x severity (1, mild; 2 , moderate; 3 , severe) and will vary from 0 to 12 points. The scale score ranges from 0 to 360 points. The items are grouped into 9 different domains: (1) cardiovascular (items 1 and 2; score, 0 to 24); (2) sleep/fatigue (items 3, 4, 5, and 6; score, 0 to 48); (3) depression/apathy (items 7, 8, 9, 10, 11, and 12; score, 0 to 72); (4) perceptual problems/hallucinations (items 13, 14, and 15; score, 0 to 36 ); (5) attention/memory (items 16, 17, and 18; score, 0 to 36); (6) gastrointestinal tract (items 19, 20, and 21; score, 0 to 36 ); (7) urinary symptoms (items 22, 23, and 24; score, 0 to 36); (8) sexual dysfunction (items 25 and
26; score, 0 to 24); (9) miscellaneous (items 27, 28, 29, and 30; score, 0 to 48 ).

Three different instruments were used to assess QoL: (1) the PDQ-39 [14]; (2) a rating of global perceived QoL (PQ$10)$ on a scale from 0 (worst) to 10 (best) $[8,15]$; and (3) the EUROHIS-QOL8 [16]. The PDQ-39 is a PD-specific questionnaire that assesses the patients' health-related QoL. There are 39 items grouped into 8 domains: (1) mobility (items 1 to 10); (2) Activities of Daily Living (items 11 to 16); (3) emotional well-being (items 17 to 22); (4) stigma (items 23 to 26); (5) social support (items 27 to 29); (6) cognition (items 30 to 33); (7) communication (items 34 to 36); and (8) pain and discomfort (items 37 to 39 ). For each item, the score may range from 0 (never) to 4 (always). The symptoms refer to the 4 weeks prior to assessment. Domain total scores are expressed as a percentage of the corresponding maximum possible score, and a Summary Index is obtained as average of the domain scores. The EUROHIS-QOL8 is an 8item global QoL questionnaire (quality of life, health status, energy, autonomy for Activities of Daily Living, self-esteem, social relationships, economic capacity, and habitat) derived from the WHOQOL-BREF. For each item, the score ranges from 0 (not at all) to 5 (completely). The total score is expressed as the mean of the individual scores. A higher score indicates a better QoL. The Schwab and England Activities of Daily Living Scale (ADLS) was used for assessing disability [17]. Functional dependency was defined as an ADLS score less than $80 \%(80 \%=$ completely independent; $70 \%=$ not completely independent) [18].

2.1. Data analysis. Data were processed using SPSS 20.0 for Windows. NMS burden was defined as follows: mild (NMSS 1-20); moderate (NMSS 21-40); severe (NMSS 41-70); and very severe (NMSS > 70) [10]. Each domain of the NMSS was expressed as a percentage: (score/total score) $\times 100$. The patients were classified according to $\mathrm{H} \& \mathrm{Y}-\mathrm{OFF}$ (1, stage I; 2 , stage II; 3, stage III; 4, stage IV /V) and NMS burden (A: 020; B: 21-40; C: 41-70; D: $\geq 71$ ) in 16 stages (HY.NMSB): $1 \mathrm{~A}$, $1 \mathrm{~B}, 1 \mathrm{C}, 1 \mathrm{D}, 2 \mathrm{~A}, 2 \mathrm{~B}, 2 \mathrm{C}, 2 \mathrm{D}, 3 \mathrm{~A}, 3 \mathrm{~B}, 3 \mathrm{C}, 3 \mathrm{D}, 4 \mathrm{~A}, 4 \mathrm{~B}, 4 \mathrm{C}$, and 4D. PDQ-39 was expressed as a Summary Index (PDQ39SI): $($ score/156) $\times 100$. For comparisons between patients with a different H\&Y stage, NMS burden stage, and/or HY.NMSB stage, chi-squared, ANOVA, and/or Mann-Whitney-Wilcoxon test were applied. With the aim of determining if the HY.NMSB contributes to the patient's QoL independently of other factors, a multiple regression analysis was conducted (PDQ-39SI as dependent variable). A $p$ value $<0.05$ was considered significant.

2.2. Standard Protocol Approvals, Registrations, and Patient Consent. For this study, we received approval from the Comité de Ética de la Investigación Clínica de Galicia from Spain (2014/534; 02/DEC/2014). Written informed consents from all participants in this study were obtained before the start of the study. COPPADIS-2015 was classified by the AEMPS (Agencia Española del Medicamento y Productos Sanitarios) as a postauthorization prospective follow-up study with the code COH-PAK-2014-01. 


\section{Results}

A total of 603 PD patients $(62.7 \pm 8.9$ years old; $59.5 \%$ males $)$ from the COPPADIS cohort were included in the analysis. The mean disease duration was $5.7 \pm 4.5$ years. One-hundred and twenty-eight (22.9\%) patients were in stage I of H\&Y, $407(67.5 \%)$ in stage II of H\&Y, $49(8.1 \%)$ in stage III of $\mathrm{H} \& \mathrm{Y}$, and only $9(1.5 \%)$ in stage IV/V of H\&Y. The mean NMSS total score was $46.7 \pm 38.2$, presenting $162(26.9 \%)$ patients with mild NMS burden, 174 (28.8\%) with moderate NMS burden, 140 (23.2\%) with severe NMS burden, and 127 (21.1\%) with very severe NMS burden. No patient presented absence of nonmotor symptoms $(\mathrm{NMSS}=0)$. Data about PD-related variables are shown in Table SM 1. When H\&Y and NMS burden were combined (HY.NMSB), a higher percentage of patients with severe or very severe NMS burden in advanced H\&Y stages (III and/or IV/V) $(p<0.0001)$ was observed (Figure 1$)$.

A worse QoL and a greater disability were associated with a higher H\&Y stage. Specifically, the PDQ-39SI and the EUROHIS-QOL8 total score were significantly lower and higher, respectively, in patients with a lower H\&Y (Table 1 and Figure 2(a)). The ADLS score was higher (indicative of lower disability) in patients with a lower H\&Y (Table 1). When patients with a consecutive stage of $H \& Y$ were compared, the most significant differences were observed between patients with a stage II of H\&Y and those ones with stage III, but no differences were observed between patients with a stage III of H\&Y and those ones with stage IV (only 9 patients in this last subgroup) (Table 1). QoL and disability were related to NMS burden as well, so the higher the NMS burden stage, the worse the QoL, and the greater the disability (Table 2 and Figure 2(b)). After classifying participants by combining both scales, H\&Y and NMSS (NMSB), QoL and disability were related to the HY.NMSB stage (Figure 2(c)): PDQ-39SI, from $6.7 \pm 4.9$ (HY.NMSB 1A) to $42.9 \pm 11.9$ (HY.NMSB 4D) $(p<0.0001)$; EUROHIS-QOL8 total score, from $4.1 \pm 0.5$ (HY.NMSB 1A) to $3.1 \pm 0.6$ (HY.NMSB 3D) $(p<0.0001$; only 1 patient in the stage $4 \mathrm{~B}$ but with a score of 4.5); and ADLS score, from 94.9 \pm 5.7 (HY.NMSB 1A) to $55 \pm 19.1$ (HY.NMSB 4D) $(p<0.0001)$. With regard to our hypothesis, it was observed that patients with a lower stage of H\&Y could have a worse QoL and/or a greater disability if they had a greater NMS burden (Tables 3 and 4). For example, patients with stage I of H\&Y and very severe NMS burden (HY.NMSB 1D; $n=15$ ) compared to patients with stage II of H\&Y but mild NMS burden (HY.NMSB 2A; $n=101)$ had a higher PDQ-39SI (28.6 \pm 17.1 vs $7.9 \pm 5.8 ; p<0.0001)$ and a lower PQ-10 (6.4 \pm 1.5 vs $7.9 \pm 1.2 ; \quad p<0.0001), \quad$ EUROHIS-QOL8 $\quad(3.5 \pm 0.4 \quad$ vs $4.1 \pm 0.4 p<0.0001)$, and ADLS score $(88 \pm 6.8$ vs $91.8 \pm 5.9$; $p=0.025$ ) (Table 3 and Figure 2). Even PDQ-39SI $(198 \pm 11.9$ vs $13.8 \pm 9.8 ; p=0.003)$ and EUROHIS-QOL8 score $(3.6 \pm 0.5$ vs $3.9 \pm 0.5 ; p=0.030)$, we are significantly higher and lower, respectively, in those patients with stage I of H\&Y and severe NMS burden HY.NMSB $1 C ; n=27$ ) than those in ones with stage $<$ II of $H \& Y$ and moderate NMS burden (HY.NMS burden 2B; $n=125$ ) (Table 3 and Figure 2). When patients with a stage II of $\mathrm{H} \& \mathrm{Y}$ were compared with those ones with a stage III, a worse QoL was observed in patients with stage II and very severe NMS burden (HY.NMSB 2D; $n=91$ ) than those in patients with a stage III of H\&Y but mild NMS burden (HY.NMSB 3A; $n=6)$ or moderate NMS burde $n=($ HY.NMSB $3 \mathrm{~B} ; n=9)$ : PDQ-39SI $31.8 \pm 3.8$ vs $14.2 \pm 10.9 p=0.003 ; 31.8 \pm 13.8$ vs. $21.5 \pm 7.9 \quad(p=0.029): \quad \mathrm{PQ}-10, \quad 6.2 \pm 1.6 \quad$ vs $\quad 8.5 \pm 1.5$ $(p=0.003) ; \quad$ EUROHIS-QOL8, $3.8 \pm 0.6$ vs $3.6 \pm 0.4$ $(p=0.048)$ (Table 4 and Figure 2 ).

In a simple linear regression model, the HY.NMSB scale predicted the PDQ-39SI: $\beta=0.480$; CI 95\%, $1.981-2.661$; $p<0.0001$. After adjustment to other covariates (age, gender, disease duration, levodopa equivalent daily dose, UPDRS-IV, FOGQ, and BDI-II), the HY.NMSB stage contributed significantly to the patient's QoL (PDQ-39SI as dependent variable) as well: adjusted $R$-squared $0.591 ; \beta=$ 0.089; CI 95\%, $0.098-0.770 ; p=0.011$ (Table 2. SM). As compared to the classical H\&Y stage alone (not significant in the model), the HY.NMS was multiplied by 12.7 the size effect over the PDQ-39SI ( $\beta$ standardized coefficient of 0.007 for the $H \& Y$ in a model with age, gender, disease duration, levodopa equivalent daily dose, UPDRS-IV, FOGQ, BDI-II, and NMSS $(p=0.823)$ vs 0.089 for the HY.NMS in the model with the same covariates included except the NMSS $(p=0.011))$.

\section{Discussion}

The present study observed that the use in PD patients of a scale that combines the H\&Y stage with the NMSS (HY.NMSB) could be useful since it would not only inform about motor and nonmotor aspects but would also serve to know how is the patient's QoL and autonomy for ADL. This is relevant because many PD patients can be in stages I to III of H\&Y for many years and stratification regarding NMS burden providing useful information not only for diagnosis but also for monitoring the outcome and ideally the response to a medication.

Ray Chaudhuri et al. [11] proposed a new strategy for clinical classification of PD patients using the NMSS in 5 stratified levels of burden ( $0=$ no NMS; $1=$ NMSS, $1-20$; $2=$ NMSS, 21-40; $3=$ NMSS, 41-70; $4=$ NMSS $>70)$ and suggested that this simple assessment could be added to existing motor-based staging (i.e., $\mathrm{H} \& \mathrm{Y}$ ) to complement PD assessment and avoid overlooking the weight of the NMS. In 951 PD patients, these authors observed a significant influence of NMS burden on disability and QoL, highlighting the need to include an NMS evaluation for a complete assessment of PD patients. We observed the same in $603 \mathrm{PD}$ patients from the COPPADIS cohort. However, here, we define specifically a scale (HY.NMSB) combining the H\&Y stage with the NMS burden: firstly, a number for the H\&Y from 1 (stage I) to 4 (stage IV/V); secondly, a letter for the NMS burden from A (non NMS or mild NMS burden; NMSS 0-20) to D (very severe burden; NMSS > 70). Combining the number with the letter, a total of 16 stages are defined, from HY.NMSB 1A (H\&Y I and non-NMS/mild NMS burden) to $4 \mathrm{D}$ (H\&Y IV/V and very severe NMS burden). PD patients without NMS (i.e., NMSS total 


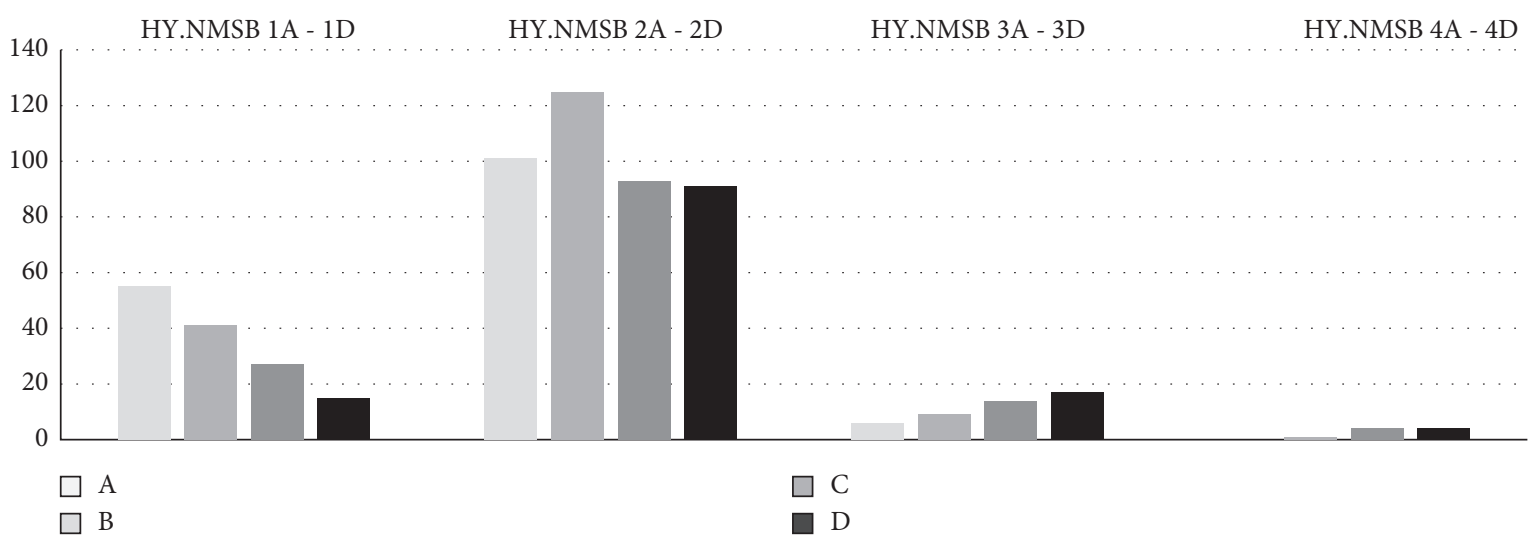

(a)
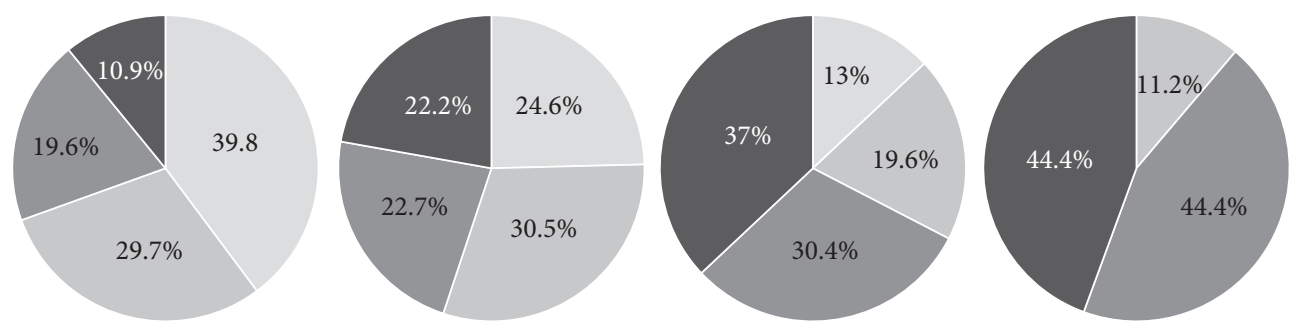

(b)

Figure 1: Total number (a) and percentage (b) of PD patients presenting with different stages of the HY.NMSB scale (from 1A to 4D) $(n=603)$.

TABLE 1: Quality of life (PDQ-39SI and EUROHIS-QOL8) and disability (ADLS score) in PD patients with regard to Hoehn and Yahr stage $(s=603)$.

\begin{tabular}{|c|c|c|c|c|c|c|c|c|}
\hline & $\mathrm{H} \& \mathrm{Y} 1, N=138$ & $\mathrm{H} \& \mathrm{Y} 2, N=407$ & $\mathrm{H} \& \mathrm{Y} 3, N=49$ & $\mathrm{H} \& \mathrm{Y} 4, N=9$ & $p^{a}$ & $p^{b}$ & $p^{c}$ & $p^{d}$ \\
\hline PDQ-39SI & $13.3 \pm 12.1$ & $17.1 \pm 12.9$ & $30.9 \pm 15.3$ & $30.3 \pm 15.3$ & $<0.0001$ & 0.037 & $<0.0001$ & 0.908 \\
\hline Mobility & $10.9 \pm 14.2$ & $16.1 \pm 18$ & $41.6 \pm 23.8$ & $37.2 \pm 24.3$ & $<0.0001$ & 0.036 & $<0.0001$ & 0.613 \\
\hline Activities of Daily Living & $13.4 \pm 14.3$ & $18.3 \pm 18.1$ & $33.6 \pm 24$ & $29.1 \pm 19.1$ & $<0.0001$ & 0.023 & $<0.0001$ & 0.604 \\
\hline Emotional well-being & $18.9 \pm 16.9$ & $21.6 \pm 20.7$ & $34.3 \pm 21.9$ & $32.8 \pm 13.2$ & $<0.0001$ & 0.372 & $<0.0001$ & 0.846 \\
\hline Stigma & $12.3 \pm 17$ & $13.2 \pm 19.7$ & $16.1 \pm 22.1$ & $22.9 \pm 32.7$ & 0.316 & 0.839 & 0.341 & 0.432 \\
\hline Social support & $6.8 \pm 14.3$ & $8.6 \pm 16.9$ & $9.7 \pm 19.7$ & $12.9 \pm 22.1$ & 0.497 & 0.492 & 0.686 & 0.656 \\
\hline Cognition & $14.9 \pm 16.4$ & $20.1 \pm 18.1$ & $27.2 \pm 18.9$ & $31.2 \pm 17.9$ & $<0.0001$ & 0.015 & 0.011 & 0.553 \\
\hline Communication & $8.8 \pm 13.8$ & $9.5 \pm 14.2$ & $17.3 \pm 19.7$ & $19.4 \pm 17.6$ & 0.001 & 0.522 & 0.001 & 0.766 \\
\hline Pain and discomfort & $21.1 \pm 19.9$ & $26.9 \pm 22.9$ & $42.5 \pm 22.5$ & $40.7 \pm 28.9$ & $<0.0001$ & 0.065 & $<0.0001$ & 0.835 \\
\hline PQ-10 & $7.5 \pm 1.5$ & $7.2 \pm 6.2$ & $6.2 \pm 2.1$ & $6.9 \pm 1.4$ & $<0.0001$ & 0.234 & $<0.0001$ & 0.375 \\
\hline EUROHIS-QOL8 & $3.9 \pm 0.5$ & $3.8 \pm 0.5$ & $3.3 \pm 0.6$ & $3.7 \pm 0.5$ & $<0.0001$ & 0.117 & $<0.0001$ & 0.135 \\
\hline Quality of life & $3.9 \pm 0.7$ & $3.8 \pm 0.7$ & $3.2 \pm 0.8$ & $3.8 \pm 0.4$ & $<0.0001$ & 0.207 & $<0.0001$ & 0.063 \\
\hline Health status & $3.4 \pm 0.8$ & $3.1 \pm 0.9$ & $2.5 \pm 0.9$ & $2.9 \pm 0.8$ & $<0.0001$ & 0.012 & $<0.0001$ & 0.296 \\
\hline Energy & $3.9 \pm 0.8$ & $3.7 \pm 0.8$ & $3.2 \pm 0.8$ & $3.7 \pm 1.1$ & $<0.0001$ & 0.195 & $<0.0001$ & 0.156 \\
\hline Autonomy for ADL & $3.8 \pm 0.7$ & $3.6 \pm 0.8$ & $2.8 \pm 0.8$ & $3.1 \pm 0.8$ & $<0.0001$ & 0.023 & $<0.0001$ & 0.289 \\
\hline Self-esteem & $3.9 \pm 0.7$ & $3.8 \pm 0.8$ & $3.4 \pm 0.9$ & $3.4 \pm 0.7$ & 0.001 & 0.437 & 0.001 & 0.863 \\
\hline Social relationships & $4 \pm 0.7$ & $4.1 \pm 0.7$ & $3.7 \pm 0.8$ & $4 \pm 0.9$ & 0.021 & 0.890 & 0.004 & 0.388 \\
\hline Economic capacity & $3.9 \pm 0.7$ & $3.7 \pm 0.8$ & $3.7 \pm 1$ & $3.9 \pm 0.7$ & 0.414 & 0.819 & 0.160 & 0.546 \\
\hline Habitat & $4.3 \pm 0.7$ & $4.2 \pm 0.7$ & $4.1 \pm 0.8$ & $4.4 \pm 0.5$ & 0.287 & 0.832 & 0.112 & 0.161 \\
\hline ADLS score & $93.5 \pm 6.9$ & $87.8 \pm 9.4$ & $77.1 \pm 13.1$ & $72.5 \pm 23.8$ & $<0.0001$ & $<0.0001$ & $<0.0001$ & 0.416 \\
\hline Functional dependency (\%) & 0.7 & 8.8 & 42.9 & 37.5 & $<0.0001$ & $<0.0001$ & $<0.0001$ & 0.546 \\
\hline
\end{tabular}

Chi-squared, Mann-Whitney-Wilcoxon, and ANOVA test were applied. The results represent percentages or mean \pm SD; $p^{a}$, all groups; $p^{b}, \mathrm{H} \& \mathrm{Y} 2 \mathrm{vs} \mathrm{H} \& \mathrm{Y} 1$; $p^{c}, \mathrm{H} \& \mathrm{Y} 3$ vs H\&Y 2; $p^{d}$, H\&Y 4 vs H\&Y 3. ADL, Activities of Daily Living; ADLS, Schwab and England Activities of Daily Living Scale; EUROHIS-QOL8, EUROHIS-QOL 8-item index; H\&Y, Hoehn and Yahr; PDQ-39SI, 39-item Parkinson's Disease Quality of Life Questionnaire Summary Index.

score $=0$ ) are rare (none in this cohort), but in any case, they are included as " $\mathrm{A}$ " because there is really no difference between, for example, a patient with NMSS total score $=0$ and another one with NMSS total score $=1$ or 3 . So, " $A$ " is defined as a patient without NMS or mild NMS burden. On the other hand and with the idea of simplifying the scale, 


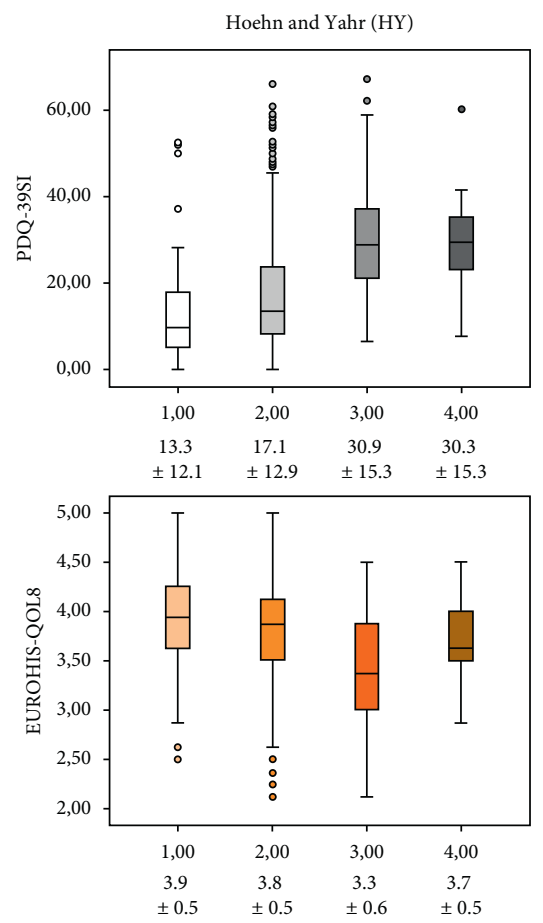

(a)
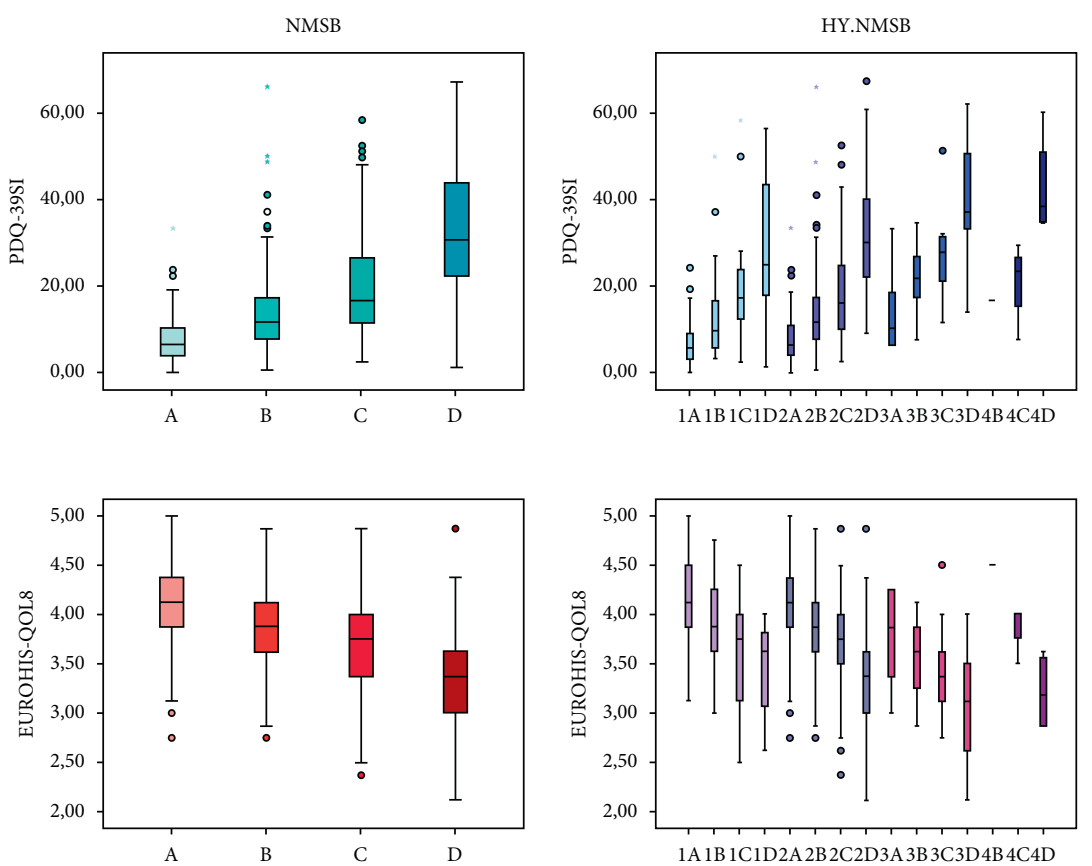

(b)

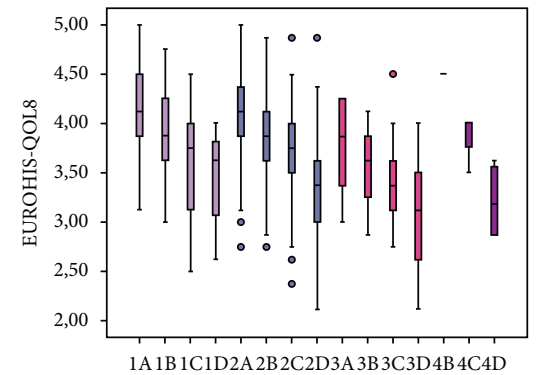

(c)

FIGURE 2: PDQ-39SI and EUROHIS-QOL8 scores in patients with regard to the H\&Y (a), the nonmotor symptoms burden (NMSB) (b), and both (HY.NMSB) (c) $(n=603)$.

TABLE 2: Quality of life (PDQ-39SI and EUROHIS-QOL8) and disability (ADLS score) in PD patients with regard to nonmotor symptoms burden: mild (NMS 1-20); moderate (NMS total score 21-40); severe (NMS total score 41-70); very severe (NMS total score > 70).

\begin{tabular}{|c|c|c|c|c|c|c|c|c|}
\hline & $\begin{array}{c}\text { Mild, } \\
N=162\end{array}$ & $\begin{array}{l}\text { Moderate, } \\
N=174\end{array}$ & $\begin{array}{l}\text { Severe, } \\
N=140\end{array}$ & $\begin{array}{c}\text { Very severe, } \\
N=127\end{array}$ & $p^{a}$ & $p^{b}$ & $p^{c}$ & $p^{d}$ \\
\hline PDQ-39SI & $7.7 \pm 5.7$ & $13.8 \pm 9.7$ & $19.9 \pm 10.7$ & $32.9 \pm 14.4$ & $<0.0001$ & $<0.0001$ & $<0.0001$ & $<0.0001$ \\
\hline Mobility & $6.2 \pm 11.5$ & $12.7 \pm 15.6$ & $19.7 \pm 15.9$ & $35.3 \pm 22.9$ & $<0.0001$ & $<0.0001$ & 0.029 & $<0.0001$ \\
\hline Activities of Daily Living & $9.5 \pm 11.1$ & $15.8 \pm 15.4$ & $19.8 \pm 16.7$ & $32.4 \pm 23.3$ & $<0.0001$ & $<0.0001$ & $<0.0001$ & $<0.0001$ \\
\hline Emotional well-being & $9.9 \pm 10.8$ & $15.6 \pm 13.9$ & $26.6 \pm 18.7$ & $41.6 \pm 22.6$ & $<0.0001$ & 0.001 & 0.149 & $<0.0001$ \\
\hline Stigma & $7.6 \pm 14.1$ & $13.7 \pm 18.9$ & $10.8 \pm 16.2$ & $22.9 \pm 25.5$ & $<0.0001$ & 0.025 & 0.001 & $<0.0001$ \\
\hline Social support & $2.2 \pm 8.9$ & $4.9 \pm 12.8$ & $10.6 \pm 17.5$ & $18.6 \pm 21.9$ & $<0.0001$ & $<0.0001$ & $<0.0001$ & 0.001 \\
\hline Cognition & $7.2 \pm 9.6$ & $16.2 \pm 13.9$ & $24.6 \pm 16.2$ & $34.9 \pm 20.1$ & $<0.0001$ & $<0.0001$ & 0.036 & $<0.0001$ \\
\hline Communication & $3.5 \pm 7.3$ & $8.3 \pm 12.4$ & $11.6 \pm 14.7$ & $19.3 \pm 19.7$ & $<0.0001$ & $<0.0001$ & $<0.0001$ & $<0.0001$ \\
\hline Pain and discomfort & $14.7 \pm 15.6$ & $20.7 \pm 18.9$ & $30.7 \pm 19.6$ & $47.4 \pm 24.8$ & $<0.0001$ & 0.002 & $<0.0001$ & $<0.0001$ \\
\hline PQ-10 & $7.9 \pm 1.2$ & $7.5 \pm 1.4$ & $6.9 \pm 1.4$ & $6.1 \pm 1.7$ & $<0.0001$ & $<0.0001$ & 0.003 & $<0.0001$ \\
\hline EUROHIS-QOL8 & $4.1 \pm 0.5$ & $3.9 \pm 0.4$ & $3.6 \pm 0.5$ & $3.3 \pm 0.6$ & $<0.0001$ & $<0.0001$ & $<0.0001$ & $<0.0001$ \\
\hline Quality of life & $4.1 \pm 0.5$ & $3.9 \pm 0.6$ & $3.7 \pm 0.7$ & $3.3 \pm 0.8$ & $<0.0001$ & 0.005 & 0.001 & $<0.0001$ \\
\hline Health status & $3.5 \pm 0.8$ & $3.3 \pm 0.8$ & $3 \pm 0.8$ & $2.6 \pm 0.9$ & $<0.0001$ & 0.001 & 0.006 & $<0.0001$ \\
\hline Energy & $4.2 \pm 0.7$ & $3.8 \pm 0.7$ & $3.6 \pm 0.8$ & $3.2 \pm 0.9$ & $<0.0001$ & $<0.0001$ & 0.004 & $<0.0001$ \\
\hline Autonomy for ADL & $4 \pm 0.7$ & $3.8 \pm 0.8$ & $3.4 \pm 0.8$ & $3 \pm 0.9$ & $<0.0001$ & 0.001 & $<0.0001$ & $<0.0001$ \\
\hline Self-esteem & $4.2 \pm 0.6$ & $3.9 \pm 0.7$ & $3.6 \pm 0.7$ & $3.3 \pm 0.9$ & $<0.0001$ & 0.010 & $<0.0001$ & 0.001 \\
\hline Social relationships & $4.4 \pm 0.6$ & $4.1 \pm 0.6$ & $3.9 \pm 0.7$ & $3.6 \pm 0.8$ & $<0.0001$ & $<0.0001$ & 0.059 & $<0.0001$ \\
\hline Economic capacity & $4.1 \pm 0.8$ & $3.9 \pm 0.7$ & $3.8 \pm 0.7$ & $3.5 \pm 0.9$ & $<0.0001$ & 0.079 & 0.180 & 0.001 \\
\hline Habitat & $4.4 \pm 0.7$ & $4.3 \pm 0.6$ & $4.2 \pm 0.7$ & $3.9 \pm 0.7$ & $<0.0001$ & 0.105 & 0.123 & 0.011 \\
\hline ADLS score & $92.9 \pm 6.1$ & $90.2 \pm 8.3$ & $86.5 \pm 10.4$ & $80.5 \pm 12.9$ & $<0.0001$ & 0.001 & $<0.0001$ & $<0.0001$ \\
\hline $\begin{array}{l}\text { Functional } \\
\text { dependency (\%) }\end{array}$ & 0.6 & 4.6 & 11.5 & 28.3 & $<0.0001$ & 0.024 & 0.019 & $<0.0001$ \\
\hline
\end{tabular}

very advanced $\mathrm{PD}$ patients with regard to motor stage (H\&Y IV and V) are considered together as number 4. After applying this scale (HY.NMSB) for the first time, we observed that QoL and disability were related to H\&Y but NMS burden as well, so patients with a lower H\&Y but a greater NMS burden can perceive a worse QoL and greater disability than patients with a higher H\&Y stage but lower NMS burden. Conventionally, H\&Y stages I and II represent mild 
TABLE 3: Quality of life (PDQ-39SI and EUROHIS-QOL8) and disability (ADLS score) in patients with stages 1C, 1D, 2A, or 2B of the HY-NMSB scale.

\begin{tabular}{lcccccccc}
\hline & $1 \mathrm{C}, N=27$ & $1 \mathrm{D}, N=15$ & $2 \mathrm{~A}, N=101$ & $2 \mathrm{~B}, N=125$ & $p^{a}$ & $p^{b}$ & $p^{c}$ & $p^{d}$ \\
\hline PDQ-39SI & $19.8 \pm 11.9$ & $28.6 \pm 17.1$ & $7.9 \pm 5.8$ & $13.8 \pm 9.8$ & $<\mathbf{0 . 0 0 0 1}$ & $<\mathbf{0 . 0 0 0 1}$ & $<\mathbf{0 . 0 0 0 1}$ & $\mathbf{0 . 0 0 3}$ \\
Mobility & $18.1 \pm 14.9$ & $27.8 \pm 17.7$ & $6.1 \pm 10.5$ & $13 \pm 16.2$ & $<\mathbf{0 . 0 0 0 1}$ & $\mathbf{0 . 0 0 1}$ & $<\mathbf{0 . 0 0 0 1}$ & $\mathbf{0 . 0 3 5}$ \\
Activities of Daily Living & $16.5 \pm 15.2$ & $28.3 \pm 18.3$ & $10.9 \pm 12.4$ & $15.9 \pm 16.1$ & $<\mathbf{0 . 0 0 0 1}$ & $\mathbf{0 . 0 0 7}$ & $\mathbf{0 . 0 5 6}$ & 0.710 \\
Emotional well-being & $28.7 \pm 16.4$ & $35.5 \pm 21.3$ & $8.9 \pm 9.4$ & $14.9 \pm 13.7$ & $<\mathbf{0 . 0 0 0 1}$ & $<\mathbf{0 . 0 0 0 1}$ & $<\mathbf{0 . 0 0 0 1}$ & $<\mathbf{0 . 0 0 0 1}$ \\
Stigma & $16.4 \pm 20.4$ & $23.8 \pm 23.9$ & $8.4 \pm 15.7$ & $13.9 \pm 19.9$ & $\mathbf{0 . 0 1 3}$ & 0.140 & $\mathbf{0 . 0 3 0}$ & 0.448 \\
Social support & $13.6 \pm 17.8$ & $19.4 \pm 22.6$ & $2.4 \pm 9.9$ & $5.3 \pm 13.8$ & $<\mathbf{0 . 0 0 0 1}$ & $\mathbf{0 . 0 0 3}$ & $<\mathbf{0 . 0 0 0 1}$ & $\mathbf{0 . 0 0 5}$ \\
Cognition & $21.9 \pm 17.4$ & $32.5 \pm 18.8$ & $8.3 \pm 9.6$ & $15.9 \pm 13.7$ & $<\mathbf{0 . 0 0 0 1}$ & $\mathbf{0 . 0 0 1}$ & $<\mathbf{0 . 0 0 0 1}$ & 0.099 \\
Communication & $14.2 \pm 15.9$ & $20.6 \pm 19.6$ & $4.1 \pm 8.4$ & $7.9 \pm 12.1$ & $<\mathbf{0 . 0 0 0 1}$ & $\mathbf{0 . 0 0 7}$ & $<\mathbf{0 . 0 0 0 1}$ & $\mathbf{0 . 0 2 6}$ \\
Pain and discomfort & $27.8 \pm 20.1$ & $38.3 \pm 27.4$ & $13.5 \pm 13.5$ & $21.2 \pm 20.3$ & $<\mathbf{0 . 0 0 0 1}$ & $\mathbf{0 . 0 1 0}$ & $<\mathbf{0 . 0 0 0 1}$ & 0.053 \\
PQ-10 & $6.9 \pm 1.6$ & $6.4 \pm 1.5$ & $7.9 \pm 1.2$ & $7.5 \pm 1.5$ & $<\mathbf{0 . 0 0 0 1}$ & $\mathbf{0 . 0 0 8}$ & $\mathbf{0 . 0 0 8}$ & 0.201 \\
EUROHIS-QOL8 & $3.6 \pm 0.5$ & $3.5 \pm 0.4$ & $4.1 \pm 0.4$ & $3.9 \pm 0.5$ & $<\mathbf{0 . 0 0 0 1}$ & $\mathbf{0 . 0 0 4}$ & $<\mathbf{0 . 0 0 0 1}$ & $\mathbf{0 . 0 3 0}$ \\
Quality of life & $3.6 \pm 0.6$ & $3.2 \pm 0.6$ & $4.1 \pm 0.6$ & $3.9 \pm 0.6$ & $<\mathbf{0 . 0 0 0 1}$ & $<\mathbf{0 . 0 0 0 1}$ & $\mathbf{0 . 0 0 1}$ & $\mathbf{0 . 0 4 8}$ \\
Health status & $3.2 \pm 0.9$ & $3.2 \pm 0.6$ & $3.6 \pm 0.7$ & $3.3 \pm 0.8$ & $\mathbf{0 . 0 1 4}$ & 0.726 & 0.051 & 0.888 \\
Energy & $3.4 \pm 0.7$ & $3.4 \pm 0.6$ & $4.1 \pm 0.6$ & $3.8 \pm 0.7$ & $<\mathbf{0 . 0 0 0 1}$ & $\mathbf{0 . 0 2 5}$ & $<\mathbf{0 . 0 0 0 1}$ & $\mathbf{0 . 0 0 9}$ \\
Autonomy for ADL & $3.5 \pm 0.7$ & $3.5 \pm 0.5$ & $4.1 \pm 0.6$ & $3.7 \pm 0.7$ & $<\mathbf{0 . 0 0 0 1}$ & 0.065 & $<\mathbf{0 . 0 0 0 1}$ & 0.089 \\
Self-esteem & $3.6 \pm 0.8$ & $3.5 \pm 0.6$ & $4.2 \pm 0.6$ & $3.9 \pm 0.7$ & $<\mathbf{0 . 0 0 0 1}$ & $\mathbf{0 . 0 1 5}$ & $<\mathbf{0 . 0 0 0 1}$ & $\mathbf{0 . 0 1 6}$ \\
Social relationships & $3.9 \pm 0.6$ & $3.8 \pm 0.6$ & $4.4 \pm 0.6$ & $4.2 \pm 0.6$ & $\mathbf{0 . 0 0 1}$ & $\mathbf{0 . 0 2 3}$ & $<\mathbf{0 . 0 0 0 1}$ & $\mathbf{0 . 0 2 2}$ \\
Economic capacity & $3.7 \pm 0.7$ & $3.4 \pm 0.7$ & $3.9 \pm 0.8$ & $3.9 \pm 0.6$ & $\mathbf{0 . 0 1 0}$ & $\mathbf{0 . 0 2 5}$ & $\mathbf{0 . 0 1 4}$ & 0.054 \\
Habitat & $3.9 \pm 0.8$ & $3.9 \pm 0.7$ & $4.4 \pm 0.6$ & $4.3 \pm 0.6$ & $\mathbf{0 . 0 0 5}$ & $\mathbf{0 . 0 0 1}$ & 0.160 & 0.114 \\
ADLS score & $92.6 \pm 7.1$ & $88 \pm 6.8$ & $91.8 \pm 5.9$ & $89.5 \pm 8$ & $\mathbf{0 . 0 2 5}$ & 0.246 & 0.461 & 0.061 \\
FD (\%) & 0 & 0 & 1 & 4 & 0.871 & 0.562 & 0.789 & 0.371 \\
\hline
\end{tabular}

Chi-squared and Mann-Whitney-Wilcoxon test were applied. The results represent percentages or mean \pm SD; $p^{a}, 1 \mathrm{D}$ vs $2 \mathrm{~A} ; p^{b}, 1 \mathrm{D}$ vs $2 \mathrm{~B} ; p^{c}, 1 \mathrm{C}$ vs $2 \mathrm{~A} ; p^{d}, 1 \mathrm{C}$ vs 2B. ADLS, Schwab and England Activities of Daily Living Scale; EUROHIS-QOL8, EUROHIS-QOL 8-item index; PDQ-39SI, 39-item Parkinson's Disease Quality of Life Questionnaire Summary Index.

TABLE 4: Quality of life (PDQ-39SI and EUROHIS-QOL8) and disability (ADLS score) in patients with stages 2C, 2D, 3A, or 3B of the HYNMSB scale.

\begin{tabular}{|c|c|c|c|c|c|c|c|c|}
\hline & $2 \mathrm{C}, N=93$ & $2 \mathrm{D}, N=91$ & $3 \mathrm{~A}, N=6$ & $3 \mathrm{~B}, N=9$ & $p^{a}$ & $p^{b}$ & $p^{c}$ & $p^{d}$ \\
\hline PDQ-39SI & $18.5 \pm 10.2$ & $31.8 \pm 13.8$ & $14.2 \pm 10.9$ & $21.5 \pm 7.9$ & 0.003 & 0.029 & 0.173 & 0.238 \\
\hline Mobility & $17.2 \pm 14.9$ & $32 \pm 2.1$ & $27.1 \pm 31.2$ & $27.5 \pm 16.3$ & 0.372 & 0.722 & 0.713 & 0.048 \\
\hline Activities of Daily Living & $18.4 \pm 16.4$ & $31.1 \pm 23.3$ & $6.9 \pm 7.7$ & $25.9 \pm 11.7$ & 0.005 & 0.722 & 0.081 & 0.084 \\
\hline Emotional well-being & $25.6 \pm 20.1$ & $41.7 \pm 23.1$ & $15.9 \pm 20.8$ & $24.5 \pm 19.5$ & 0.014 & 0.036 & 0.203 & 0.953 \\
\hline Stigma & $9.3 \pm 15.1$ & $22.4 \pm 24.4$ & $7.3 \pm 15$ & $6.9 \pm 14.1$ & 0.109 & 0.073 & 0.636 & 0.749 \\
\hline Social support & $9.9 \pm 16.7$ & $19.1 \pm 21.9$ & $0 \pm 0$ & $2.8 \pm 4.2$ & 0.015 & 0.048 & 0.081 & 0.526 \\
\hline Cognition & $24.4 \pm 15.9$ & $35.7 \pm 21.2$ & $1 \pm 2.6$ & $24.3 \pm 13.4$ & $<0.0001$ & 0.109 & $<0.0001$ & 0.948 \\
\hline Communication & $9.5 \pm 13.6$ & $18.4 \pm 18.7$ & $2.8 \pm 6.8$ & $13.9 \pm 13.8$ & 0.025 & 0.633 & 0.183 & 0.226 \\
\hline Pain and discomfort & $30.9 \pm 19.6$ & $46.1 \pm 24.6$ & $34.7 \pm 22.6$ & $28.7 \pm 19.6$ & 0.209 & 0.031 & 0.830 & 0.673 \\
\hline PQ-10 & $7 \pm 1.4$ & $6.2 \pm 1.6$ & $8.5 \pm 1.5$ & $7.2 \pm 1.3$ & 0.003 & 0.069 & 0.025 & 0.620 \\
\hline EUROHIS-QOL8 & $3.7 \pm 0.4$ & $3.3 \pm 0.6$ & $3.8 \pm 0.6$ & $3.6 \pm 0.4$ & 0.048 & 0.169 & 0.590 & 0.377 \\
\hline Quality of life & $3.7 \pm 0.7$ & $3.4 \pm 0.7$ & $4 \pm 0.9$ & $3.6 \pm 0.5$ & 0.108 & 0.563 & 0.462 & 0.328 \\
\hline Health status & $3 \pm 0.7$ & $2.6 \pm 0.9$ & $3.2 \pm 0.7$ & $2.7 \pm 0.5$ & 0.134 & 0.774 & 0.598 & 0.139 \\
\hline Energy & $3.7 \pm 0.7$ & $3.2 \pm 0.9$ & $3.8 \pm 0.4$ & $3.6 \pm 0.5$ & 0.078 & 0.293 & 0.553 & 0.544 \\
\hline Autonomy for ADL & $3.4 \pm 0.7$ & $3 \pm 0.9$ & $3 \pm 0.6$ & $3.2 \pm 0.7$ & 0.819 & 0.625 & 0.124 & 0.340 \\
\hline Self-esteem & $3.6 \pm 0.7$ & $3.2 \pm 0.9$ & $4 \pm 0.9$ & $3.8 \pm 0.7$ & 0.083 & 0.112 & 0.335 & 0.696 \\
\hline Social relationships & $4 \pm 0.7$ & $3.6 \pm 0.8$ & $4 \pm 0.7$ & $3.9 \pm 0.6$ & 0.115 & 0.322 & 0.649 & 0.520 \\
\hline Economic capacity & $3.9 \pm 0.7$ & $3.5 \pm 0.9$ & $4.2 \pm 0.7$ & $3.8 \pm 1.1$ & 0.064 & 0.231 & 0.311 & 0.855 \\
\hline Habitat & $4.3 \pm 0.6$ & $3.9 \pm 0.7$ & $4.2 \pm 0.7$ & $4.1 \pm 0.9$ & 0.369 & 0.255 & 0.713 & 0.751 \\
\hline ADLS score & $86.5 \pm 9.3$ & $81.8 \pm 11.5$ & $90 \pm 8.9$ & $83.3 \pm 10$ & 0.091 & 0.769 & 0.421 & 0.278 \\
\hline $\mathrm{FD}(\%)$ & 9.7 & 25.3 & 0 & 22.2 & 0.187 & 0.601 & 0.556 & 0.250 \\
\hline
\end{tabular}

Chi-squared and Mann-Whitney-Wilcoxon test were applied. The results represent percentages or mean $\pm \mathrm{SD} ; p^{a}, 2 \mathrm{D}$ vs $3 \mathrm{~A} ; p^{b}, 2 \mathrm{D}$ vs $3 \mathrm{~B} ; p^{c}, 2 \mathrm{C}$ vs $3 \mathrm{~A} ; p^{d}, 2 \mathrm{C}$ vs 3B. ADLS, Schwab and England Activities of Daily Living Scale; EUROHIS-QOL8, EUROHIS-QOL 8-item index; PDQ-39SI, 39-item Parkinson's Disease Quality of Life Questionnaire Summary Index.

$\mathrm{PD}$, but this qualification cannot be supported attending the load of NMS and any domain/s they belong. The NMS present in PD may be very variable in number and type, and they maintain only a moderate association with the motor disturbances $[10,11,19,20]$. In fact, although as expected, patients with mild NMS burden $(A ; 39.8 \%)$ were the most frequent in the group with a stage I of $H \& Y$ and patients with very severe NMS burden $(D ; 44.4 \%)$ in the group with $\mathrm{H} \& \mathrm{Y}$ IV/V; more than $30 \%$ of the patients in stage I of H\&Y had severe or very severe NMS burden. Clinical and 
TABLE 5: COPPADIS study group.

\begin{tabular}{|c|c|c|c|}
\hline $\begin{array}{l}\text { Name (last name, first } \\
\text { name) }\end{array}$ & Location & Role & Contribution \\
\hline $\begin{array}{l}\text { Astrid Adarmes, } \\
\text { Daniela }\end{array}$ & $\begin{array}{l}\text { Hospital Universitario Virgen del Rocío, Sevilla, } \\
\text { Spain }\end{array}$ & Site investigator & $\begin{array}{c}\text { Evaluation of participants and/ } \\
\text { or data management }\end{array}$ \\
\hline Almeria, Marta & $\begin{array}{l}\text { Hospital Universitari Mutua de Terrassa, Terrassa, } \\
\text { Barcelona, Spain }\end{array}$ & Site investigator & $\begin{array}{l}\text { Neuropsychologist; evaluation } \\
\text { of participants }\end{array}$ \\
\hline $\begin{array}{l}\text { Alonso Losada, Maria } \\
\text { Gema }\end{array}$ & $\begin{array}{c}\text { Hospital Álvaro Cunqueiro, Complejo } \\
\text { Hospitalario Universitario de Vigo (CHUVI), } \\
\text { Vigo, Spain }\end{array}$ & Site investigator /PI & $\begin{array}{l}\text { Coordination at the center } \\
\text { Evaluation of participants and/ } \\
\text { or data management }\end{array}$ \\
\hline $\begin{array}{l}\text { Alonso Cánovas, } \\
\text { Araceli }\end{array}$ & $\begin{array}{c}\text { Hospital Universitario Ramón y Cajal, Madrid, } \\
\text { Spain }\end{array}$ & Site investigator & $\begin{array}{c}\text { Evaluation of participants and/ } \\
\text { or data management }\end{array}$ \\
\hline $\begin{array}{l}\text { Alonso Frech, } \\
\text { Fernando }\end{array}$ & $\begin{array}{c}\text { Hospital Universitario Clínico San Carlos, } \\
\text { Madrid, Spain }\end{array}$ & Site investigator & $\begin{array}{c}\text { Evaluation of participants and/ } \\
\text { or data management }\end{array}$ \\
\hline Aneiros Díaz, Ángel & $\begin{array}{l}\text { Complejo Hospitalario Universitario de Ferrol } \\
\text { (CHUF), Ferrol, A Coruña, Spain }\end{array}$ & Site investigator /PI & $\begin{array}{l}\text { Coordination at the center } \\
\text { Evaluation of participants and/ } \\
\text { or data management }\end{array}$ \\
\hline Álvarez, Ignacio & $\begin{array}{c}\text { Hospital Universitari Mutua de Terrassa, Terrassa, } \\
\text { Barcelona, Spain }\end{array}$ & Site investigator & $\begin{array}{c}\text { Evaluation of participants and/ } \\
\text { or data management }\end{array}$ \\
\hline Álvarez Sauco, María & $\begin{array}{l}\text { Hospital General Universitario de Elche, Elche, } \\
\text { Spain }\end{array}$ & Site investigator /PI & $\begin{array}{l}\text { Coordination at the center } \\
\text { Evaluation of participants and/ } \\
\text { or data management }\end{array}$ \\
\hline Arnáiz, Sandra & $\begin{array}{c}\text { Complejo Asistencial Universitario de Burgos, } \\
\text { Burgos, Spain }\end{array}$ & Site investigator & $\begin{array}{c}\text { Evaluation of participants and/ } \\
\text { or data management }\end{array}$ \\
\hline Arribas, Sonia & $\begin{array}{c}\text { Hospital Universitari Mutua de Terrassa, Terrassa, } \\
\text { Barcelona, Spain }\end{array}$ & Site investigator & $\begin{array}{l}\text { Neuropsychologist; evaluation } \\
\text { of participants }\end{array}$ \\
\hline $\begin{array}{l}\text { Ascunce Vidondo, } \\
\text { Arancha }\end{array}$ & $\begin{array}{c}\text { Complejo Hospitalario de Navarra, Pamplona, } \\
\text { Spain }\end{array}$ & Site investigator & $\begin{array}{l}\text { Evaluation of participa } \\
\text { or data managem }\end{array}$ \\
\hline Aguilar, Miquel & $\begin{array}{c}\text { Hospital Universitari Mutua de Terrassa, Terrassa, } \\
\text { Barcelona, Spain }\end{array}$ & Site investigator & $\begin{array}{c}\text { Evaluation of participants and/ } \\
\text { or data management }\end{array}$ \\
\hline $\begin{array}{l}\text { Ávila Rivera, Maria } \\
\text { Asunción }\end{array}$ & $\begin{array}{c}\text { Consorci Sanitari Integral, Hospital General de L } \\
\text { 'Hospitalet, L'Hospitalet de Llobregat, Barcelona, } \\
\text { Spain }\end{array}$ & Site investigator /PI & $\begin{array}{l}\text { Coordination at the center } \\
\text { Evaluation of participants and/ } \\
\text { or data management }\end{array}$ \\
\hline $\begin{array}{l}\text { Bernardo Lambrich, } \\
\text { Noemí }\end{array}$ & $\begin{array}{c}\text { Hospital de Tortosa Verge de la Cinta (HTVC), } \\
\text { Tortosa, Tarragona, Spain }\end{array}$ & Site investigator & $\begin{array}{c}\text { Evaluation of participants and/ } \\
\text { or data management }\end{array}$ \\
\hline Bejr-Kasem, Helena & Hospital de Sant Pau, Barcelona, Spain & Site investigator & $\begin{array}{c}\text { Evaluation of participants and/ } \\
\text { or data management }\end{array}$ \\
\hline $\begin{array}{l}\text { Blázquez Estrada, } \\
\text { Marta }\end{array}$ & $\begin{array}{c}\text { Hospital Universitario Central de Asturias, } \\
\text { Oviedo, Spain }\end{array}$ & Site investigator & Evaluation of participants \\
\hline $\begin{array}{l}\text { Botí González, Maria } \\
\text { Ángeles }\end{array}$ & $\begin{array}{c}\text { Hospital Universitari Mutua de Terrassa, Terrassa, } \\
\text { Barcelona, Spain }\end{array}$ & Site investigator & $\begin{array}{l}\text { Neuropsychologist; evaluation } \\
\text { of participants }\end{array}$ \\
\hline Borrué, Carmen & Hospital Infanta Sofía, Madrid, Spain & Site investigator /PI & $\begin{array}{l}\text { Coordination at the center } \\
\text { Evaluation of participants and/ } \\
\text { or data management }\end{array}$ \\
\hline $\begin{array}{l}\text { Buongiorno, Maria } \\
\text { Teresa }\end{array}$ & $\begin{array}{c}\text { Hospital Universitari Mutua de Terrassa, Terrassa, } \\
\text { Barcelona, Spain }\end{array}$ & Site investigator & Nurse study coordinator \\
\hline $\begin{array}{l}\text { Cabello González, } \\
\text { Carolina }\end{array}$ & $\begin{array}{c}\text { Complejo Hospitalario de Navarra, Pamplona, } \\
\text { Spain }\end{array}$ & Site investigator & Scheduling of evaluations \\
\hline Cabo López, Iria & $\begin{array}{l}\text { Complejo Hospitalario Universitario de } \\
\text { Pontevedra (CHOP), Pontevedra, Spain }\end{array}$ & Site investigator /PI & $\begin{array}{l}\text { Coordination at the center } \\
\text { Evaluation of participants and/ } \\
\text { or data management }\end{array}$ \\
\hline Caballol, Nuria & $\begin{array}{l}\text { Consorci Sanitari Integral, Hospital Moisés } \\
\text { Broggi, Sant Joan Despí, Barcelona, Spain. }\end{array}$ & Site investigator /PI & $\begin{array}{l}\text { Coordination at the center } \\
\text { Evaluation of participants and/ } \\
\text { or data management }\end{array}$ \\
\hline Cámara Lorenzo, Ana & Hospital Clínic de Barcelona, Barcelona, Spain & Site investigator & Nurse study coordinator \\
\hline Carrillo, Fátima & $\begin{array}{l}\text { Hospital Universitario Virgen del Rocío, Sevilla, } \\
\text { Spain }\end{array}$ & Site investigator & $\begin{array}{c}\text { Evaluation of participants and/ } \\
\text { or data management }\end{array}$ \\
\hline $\begin{array}{l}\text { Carrillo Padilla, } \\
\text { Francisco José }\end{array}$ & $\begin{array}{l}\text { Hospital Universitario de Canarias, San Cristóbal } \\
\text { de la Laguna, Santa Cruz de Tenerife, Spain }\end{array}$ & Site investigator /PI & Coordination at the center \\
\hline Casas, Elena & $\begin{array}{c}\text { Complejo Asistencial Universitario de Burgos, } \\
\text { Burgos, Spain }\end{array}$ & Site investigator & $\begin{array}{c}\text { Evaluation of participants and/ } \\
\text { or data management }\end{array}$ \\
\hline
\end{tabular}


TABle 5: Continued.

\begin{tabular}{|c|c|c|c|}
\hline $\begin{array}{l}\text { Name (last name, first } \\
\text { name) }\end{array}$ & Location & Role & Contribution \\
\hline Catalán, Maria Joé & $\begin{array}{l}\text { Hospital Universitario Clínico San Carlos, } \\
\text { Madrid, Spain }\end{array}$ & Site investigator /PI & $\begin{array}{c}\text { Coordination at the center } \\
\text { Evaluation of participants and/ } \\
\text { or data management }\end{array}$ \\
\hline Clavero, Pedro & $\begin{array}{c}\text { Complejo Hospitalario de Navarra, Pamplona, } \\
\text { Spain }\end{array}$ & Site investigator & $\begin{array}{c}\text { Evaluation of participants and/ } \\
\text { or data management }\end{array}$ \\
\hline Cortina Fernández, A & $\begin{array}{l}\text { Complejo Hospitalario Universitario de Ferrol } \\
\text { (CHUF), Ferrol, A Coruña, Spain }\end{array}$ & Site investigator & $\begin{array}{l}\text { Coordination of blood } \\
\text { extractions }\end{array}$ \\
\hline Cosgaya, Marina & Hospital Clínic de Barcelona, Barcelona, Spain & Site investigator & $\begin{array}{c}\text { Evaluation of participants and/ } \\
\text { or data management }\end{array}$ \\
\hline Cots Foraster, Anna & $\begin{array}{l}\text { Institut d'Assistència Sanitària (IAS) - Instituí } \\
\text { Cátala de la Salud. Girona, Spain }\end{array}$ & Site investigator & $\begin{array}{c}\text { Evaluation of participants and/ } \\
\text { or data management }\end{array}$ \\
\hline Crespo Cuevas, Ane & Hospital del Mar, Barcelona, Spain. & Site investigator & $\begin{array}{c}\text { Evaluation of participants and/ } \\
\text { or data management }\end{array}$ \\
\hline Cubo, Esther & $\begin{array}{c}\text { Complejo Asistencial Universitario de Burgos, } \\
\text { Burgos, Spain }\end{array}$ & Site investigator /PI & $\begin{array}{c}\text { Coordination at the center } \\
\text { Evaluation of participants and/ } \\
\text { or data management }\end{array}$ \\
\hline $\begin{array}{l}\text { De Deus Fonticoba, } \\
\text { Teresa }\end{array}$ & $\begin{array}{l}\text { Complejo Hospitalario Universitario de Ferrol } \\
\text { (CHUF), Ferrol, A Coruña, Spain }\end{array}$ & Site investigator & $\begin{array}{c}\text { Nurse study coordinator } \\
\text { Evaluation of participants and/ } \\
\text { or data management }\end{array}$ \\
\hline De Fábregues, Oriol & $\begin{array}{l}\text { Hospital Universitario Vall d'Hebron, Barcelona, } \\
\text { Spain }\end{array}$ & Site investigator /PI & $\begin{array}{c}\text { Coordination at the center } \\
\text { Evaluation of participants and/ } \\
\text { or data management }\end{array}$ \\
\hline Díez Fairen, M & $\begin{array}{c}\text { Hospital Universitari Mutua de Terrassa, Terrassa, } \\
\text { Barcelona, Spain }\end{array}$ & Site investigator & $\begin{array}{c}\text { Evaluation of participants and/ } \\
\text { or data management }\end{array}$ \\
\hline Erro, Elena & $\begin{array}{l}\text { Complejo Hospitalario de Navarra, Pamplona, } \\
\text { Spain }\end{array}$ & Site investigator & $\begin{array}{c}\text { Evaluation of participants and/ } \\
\text { or data management }\end{array}$ \\
\hline Escalante, Sonia & $\begin{array}{c}\text { Hospital de Tortosa Verge de la Cinta (HTVC), } \\
\text { Tortosa, Tarragona, Spain }\end{array}$ & Site investigator /PI & $\begin{array}{c}\text { Coordination at the center } \\
\text { Evaluation of participants and/ } \\
\text { or data management }\end{array}$ \\
\hline stelrich Peyret, Elena & $\begin{array}{c}\text { Institut d'Assistència Sanitària (IAS) - Instituí } \\
\text { Cátala de la Salud. Girona, Spain }\end{array}$ & Site investigator & $\begin{array}{c}\text { Evaluation of participants and/ } \\
\text { or data management }\end{array}$ \\
\hline $\begin{array}{l}\text { Fernández Guillán, } \\
\text { Noelia }\end{array}$ & $\begin{array}{l}\text { Complejo Hospitalario Universitario de Ferrol } \\
\text { (CHUF), Ferrol, A Coruña, Spain }\end{array}$ & Site investigator & Neuroimaging studies \\
\hline Gámez, Pedro & $\begin{array}{c}\text { Complejo Asistencial Universitario de Burgos, } \\
\text { Burgos, Spain }\end{array}$ & Site investigator & $\begin{array}{c}\text { Evaluation of participants and/ } \\
\text { or data management }\end{array}$ \\
\hline Gallego, Mercedes & Hospital La Princesa, Madrid, Spain & Site investigator & $\begin{array}{c}\text { Evaluation of participants and/ } \\
\text { or data management }\end{array}$ \\
\hline García Caldentey, Juan & $\begin{array}{l}\text { Centro Neurológico Oms 42, Palma de Mallorca, } \\
\text { Spain }\end{array}$ & Site investigator /PI & $\begin{array}{l}\text { Coordination at the center } \\
\text { Evaluation of participants and/ } \\
\text { or data management }\end{array}$ \\
\hline $\begin{array}{l}\text { García Campos, } \\
\text { Cristina }\end{array}$ & $\begin{array}{l}\text { Hospital Universitario Virgen Macarena, Sevilla, } \\
\text { Spain }\end{array}$ & Site investigator & $\begin{array}{c}\text { Evaluation of participants and/ } \\
\text { or data management }\end{array}$ \\
\hline $\begin{array}{l}\text { García Moreno, Jose } \\
\text { Manuel }\end{array}$ & $\begin{array}{l}\text { Hospital Universitario Virgen Macarena, Sevilla, } \\
\text { Spain }\end{array}$ & Site investigator /PI & $\begin{array}{l}\text { Coordination at the center } \\
\text { Evaluation of participants and/ } \\
\text { or data management }\end{array}$ \\
\hline Gastón, Itziar & $\begin{array}{c}\text { Complejo Hospitalario de Navarra, Pamplona, } \\
\text { Spain }\end{array}$ & Site investigator /PI & $\begin{array}{c}\text { Coordination at the center } \\
\text { Evaluation of participants and/ } \\
\text { or data management }\end{array}$ \\
\hline Guillén Fopiani, Desiré & $\begin{array}{l}\text { Complejo Hospitalario Universitario de } \\
\text { Pontevedra (CHOP), Pontevedra, Spain }\end{array}$ & Site investigator & $\begin{array}{l}\text { Neuropsychologist; evaluation } \\
\text { of participants }\end{array}$ \\
\hline $\begin{array}{l}\text { Gómez Garre, María } \\
\text { del Pilar }\end{array}$ & $\begin{array}{c}\text { Hospital Universitario Virgen del Rocío, Sevilla, } \\
\text { Spain }\end{array}$ & Site investigator & Genetic studies coordination \\
\hline $\begin{array}{l}\text { Gómez Mayordomo, } \\
\text { Víctor }\end{array}$ & Hospital Clínico San Carlos, Madrid, Spain & Site investigator & $\begin{array}{c}\text { Evaluation of participants and/ } \\
\text { or data management }\end{array}$ \\
\hline González Aloy, Javier & $\begin{array}{l}\text { Institut d'Assistència Sanitària (IAS) - Instituí } \\
\text { Cátala de la Salud. Girona, Spain }\end{array}$ & Site investigator & $\begin{array}{c}\text { Evaluation of participants and/ } \\
\text { or data management }\end{array}$ \\
\hline $\begin{array}{l}\text { González Aramburu, } \\
\text { Isabel }\end{array}$ & $\begin{array}{c}\text { Hospital Universitario Marqués de Valdecilla, } \\
\text { Santander, Spain }\end{array}$ & Site investigator & $\begin{array}{c}\text { Evaluation of participants and/ } \\
\text { or data management }\end{array}$ \\
\hline
\end{tabular}


TABle 5: Continued.

\begin{tabular}{|c|c|c|c|}
\hline $\begin{array}{l}\text { Name (last name, first } \\
\text { name) }\end{array}$ & Location & Role & Contribution \\
\hline $\begin{array}{l}\text { González Ardura, } \\
\text { Jessica }\end{array}$ & $\begin{array}{l}\text { Hospital Universitario Lucus Augusti (HULA), } \\
\text { Lugo, Spain }\end{array}$ & Site investigator /PI & $\begin{array}{c}\text { Coordination at the center } \\
\text { Evaluation of participants and/ } \\
\text { or data management }\end{array}$ \\
\hline $\begin{array}{l}\text { González García, } \\
\text { Beatriz }\end{array}$ & Hospital La Princesa, Madrid, Spain & Site investigator & Nurse study coordinator \\
\hline $\begin{array}{l}\text { González Palmás, } \\
\text { Maria Josefa }\end{array}$ & $\begin{array}{l}\text { Complejo Hospitalario Universitario de } \\
\text { Pontevedra (CHOP), Pontevedra, Spain }\end{array}$ & Site investigator & $\begin{array}{c}\text { Evaluation of participants and/ } \\
\text { or data management }\end{array}$ \\
\hline $\begin{array}{l}\text { González Toledo, } \\
\text { Gabriel Ricardo }\end{array}$ & $\begin{array}{l}\text { Hospital Universitario de Canarias, San Cristóbal } \\
\text { de la Laguna, Santa Cruz de Tenerife, Spain }\end{array}$ & Site investigator & $\begin{array}{c}\text { Evaluation of participants and/ } \\
\text { or data management }\end{array}$ \\
\hline Golpe Díaz, Ana & $\begin{array}{l}\text { Complejo Hospitalario Universitario de Ferrol } \\
\text { (CHUF), Ferrol, A Coruña, Spain }\end{array}$ & Site investigator & $\begin{array}{l}\text { Laboratory analysis } \\
\text { coordination }\end{array}$ \\
\hline Grau Solá, Mireia & $\begin{array}{c}\text { Consorci Sanitari Integral, Hospital Moisés } \\
\text { Broggi, Sant Joan Despí, Barcelona, Spain }\end{array}$ & Site investigator & $\begin{array}{c}\text { Evaluation of participants and/ } \\
\text { or data management }\end{array}$ \\
\hline Guardia, Gemma & $\begin{array}{c}\text { Hospital Universitari Mutua de Terrassa, Terrassa, } \\
\text { Barcelona, Spain }\end{array}$ & Site investigator & $\begin{array}{c}\text { Evaluation of participants and/ } \\
\text { or data management }\end{array}$ \\
\hline Hernández Vara, Jorge & $\begin{array}{l}\text { Hospital Universitario Vall d'Hebron, Barcelona, } \\
\text { Spain }\end{array}$ & Site investigator /PI & $\begin{array}{l}\text { Coordination at the center } \\
\text { Evaluation of participants and/ } \\
\text { or data management }\end{array}$ \\
\hline Horta Barba, Andrea & Hospital de Sant Pau, Barcelona, Spain & Site investigator & $\begin{array}{l}\text { Neuropsychologist; evaluation } \\
\text { of participants }\end{array}$ \\
\hline $\begin{array}{l}\text { Idoate Calderón, } \\
\text { Daniel }\end{array}$ & $\begin{array}{l}\text { Complejo Hospitalario Universitario de } \\
\text { Pontevedra (CHOP), Pontevedra, Spain }\end{array}$ & Site investigaor & $\begin{array}{l}\text { Neuropsychologist; evaluation } \\
\text { of participants }\end{array}$ \\
\hline Infante, Jon & $\begin{array}{c}\text { Hospital Universitario Marqués de Valdecilla, } \\
\text { Santander, Spain }\end{array}$ & Site investigator /PI & $\begin{array}{l}\text { Coordination at the center } \\
\text { Evaluation of participants and/ } \\
\text { or data management }\end{array}$ \\
\hline Jesús, Silvia & $\begin{array}{l}\text { Hospital Universitario Virgen del Rocío, Sevilla, } \\
\text { Spain }\end{array}$ & Site investigator & $\begin{array}{c}\text { Evaluation of participants and/ } \\
\text { or data management } \\
\text { Coordination at the center }\end{array}$ \\
\hline Kulievsky, Jaime & Hospital de Sant Pau, Barcelona, Spain & Site investigator /PI & $\begin{array}{c}\text { Evaluation of participants and/ } \\
\text { or data management } \\
\text { Coordination at the center }\end{array}$ \\
\hline Kurtis, Mónica & Hospital Ruber Internacional, Madrid, Spain & Site investigator /PI & $\begin{array}{c}\text { Evaluation of participants and/ } \\
\text { or data management }\end{array}$ \\
\hline Labandeira, Carmen & $\begin{array}{c}\text { Hospital Álvaro Cunqueiro, Complejo } \\
\text { Hospitalario Universitario de Vigo (CHUVI), } \\
\text { Vigo, Spain }\end{array}$ & Site investigator & $\begin{array}{l}\text { Evaluation of participants and/ } \\
\text { or data management }\end{array}$ \\
\hline $\begin{array}{l}\text { Labrador Espinosa, } \\
\text { Miguel Ángel }\end{array}$ & $\begin{array}{l}\text { Hospital Universitario Virgen del Rocío, Sevilla, } \\
\text { Spain }\end{array}$ & Site investigator & Neuroimaging data analysis \\
\hline Lacruz, Francisco & $\begin{array}{c}\text { Complejo Hospitalario de Navarra, Pamplona, } \\
\text { Spain }\end{array}$ & Site investigator & $\begin{array}{c}\text { Evaluation of participants and/ } \\
\text { or data management }\end{array}$ \\
\hline Lage Castro, Melva & $\begin{array}{l}\text { Complejo Hospitalario Universitario de } \\
\text { Pontevedra (CHOP), Pontevedra, Spain }\end{array}$ & Site investigator & $\begin{array}{l}\text { Evaluation of participants and/ } \\
\text { or data management }\end{array}$ \\
\hline Legarda, Inés & $\begin{array}{c}\text { Hospital Universitario Son Espases, Palma de } \\
\text { Mallorca, Spain }\end{array}$ & Site investigator /PI & $\begin{array}{l}\text { Coordination at the center } \\
\text { Evaluation of participants and/ } \\
\text { or data management }\end{array}$ \\
\hline López Ariztegui, Nuria & Complejo Hospitalario de Toledo, Toledo, Spain & Site investigator /PI & $\begin{array}{c}\text { Evaluation of participants and/ } \\
\text { or data management }\end{array}$ \\
\hline $\begin{array}{l}\text { López Díaz, Luis } \\
\text { Manuel }\end{array}$ & Hospital Da Costa de Burela, Lugo, Spain & Site investigator & $\begin{array}{c}\text { Evaluation of participants and/ } \\
\text { or data management }\end{array}$ \\
\hline $\begin{array}{l}\text { López Manzanares, } \\
\text { Lydia }\end{array}$ & Hospital La Princesa, Madrid, Spain & Site investigator /PI & $\begin{array}{l}\text { Coordination at the center } \\
\text { Evaluation of participants and/ } \\
\text { or data management }\end{array}$ \\
\hline López Seoane, Balbino & $\begin{array}{l}\text { Complejo Hospitalario Universitario de Ferrol } \\
\text { (CHUF), Ferrol, A Coruña, Spain }\end{array}$ & Site investigator & Neuroimaging studies \\
\hline Lucas del Pozo, Sara & $\begin{array}{l}\text { Hospital Universitario Vall d'Hebron, Barcelona, } \\
\text { Spain }\end{array}$ & Site investigator & $\begin{array}{l}\text { Evaluation of participants and/ } \\
\text { or data management }\end{array}$ \\
\hline Macías, Yolanda & Fundación Hospital de Alcorcón, Madrid, Spain & Site investigator & $\begin{array}{c}\text { Evaluation of participants and/ } \\
\text { or data management }\end{array}$ \\
\hline
\end{tabular}


TABle 5: Continued.

\begin{tabular}{|c|c|c|c|}
\hline $\begin{array}{l}\text { Name (last name, first } \\
\text { name) }\end{array}$ & Location & Role & Contribution \\
\hline Mata, Marina & Hospital Infanta Sofía, Madrid, Spain & Site investigator & $\begin{array}{c}\text { Evaluation of participants and/ } \\
\text { or data management }\end{array}$ \\
\hline Martí Andres, Gloria & $\begin{array}{l}\text { Hospital Universitario Vall d'Hebron, Barcelona, } \\
\text { Spain }\end{array}$ & Site investigator & $\begin{array}{c}\text { Evaluation of participants and/ } \\
\text { or data management } \\
\text { Coordination at the center }\end{array}$ \\
\hline Martí, Maria José & Hospital Clínic de Barcelona, Barcelona, Spain & Site investigator /PI & $\begin{array}{c}\text { Evaluation of participants and/ } \\
\text { or data management }\end{array}$ \\
\hline $\begin{array}{l}\text { Martínez Castrillo, } \\
\text { Juan Carlos }\end{array}$ & $\begin{array}{l}\text { Hospital Universitario Ramón y Cajal, Madrid, } \\
\text { Spain }\end{array}$ & Site investigator /PI & $\begin{array}{l}\text { Coordination at the center } \\
\text { Evaluation of participants and/ } \\
\text { or data management }\end{array}$ \\
\hline $\begin{array}{l}\text { Martinez-Martin, } \\
\text { Pablo }\end{array}$ & $\begin{array}{l}\text { Centro Nacional de Epidemiología y CIBERNED, } \\
\text { Instituto de Salud Carlos III. Madrid }\end{array}$ & $\begin{array}{l}\text { Collaborator in statistical } \\
\text { and methods analysis }\end{array}$ & Methods and statistical reviewer \\
\hline McAfee, Darrian & University of Pennsylvania, Philadelphia & $\begin{array}{l}\text { Collaborator in english } \\
\text { style }\end{array}$ & English style reviewer \\
\hline Meitín, Maria Teresa & Hospital Da Costa de Burela, Lugo, Spain & Site investigator & $\begin{array}{c}\text { Evaluation of participants and/ } \\
\text { or data management }\end{array}$ \\
\hline $\begin{array}{l}\text { Menéndez González, } \\
\text { Manuel }\end{array}$ & $\begin{array}{c}\text { Hospital Universitario Central de Asturias, } \\
\text { Oviedo, Spain }\end{array}$ & Site investigator /PI & $\begin{array}{c}\text { Coordination at the center } \\
\text { Evaluation of participants and/ } \\
\text { or data management }\end{array}$ \\
\hline $\begin{array}{l}\text { Méndez del Barrio, } \\
\text { Carlota }\end{array}$ & $\begin{array}{l}\text { Hospital Universitario Virgen del Rocío, Sevilla, } \\
\text { Spain }\end{array}$ & Site investigator & $\begin{array}{c}\text { Evaluation of participants and/ } \\
\text { or data management }\end{array}$ \\
\hline Mir, Pablo & $\begin{array}{l}\text { Hospital Universitario Virgen del Rocío, Sevilla, } \\
\text { Spain }\end{array}$ & Site investigator /PI & $\begin{array}{c}\text { Coordination at the center } \\
\text { Evaluation of participants and/ } \\
\text { or data management }\end{array}$ \\
\hline $\begin{array}{l}\text { Miranda Santiago, } \\
\text { Javier }\end{array}$ & $\begin{array}{c}\text { Complejo Asistencial Universitario de Burgos, } \\
\text { Burgos, Spain }\end{array}$ & Site investigator & $\begin{array}{l}\text { Evaluation of participants and/ } \\
\text { or data management }\end{array}$ \\
\hline $\begin{array}{l}\text { Morales Casado, Maria } \\
\text { Isabel }\end{array}$ & Complejo Hospitalario de Toledo, Toledo, Spain. & Site investigator & $\begin{array}{l}\text { Evaluation of participants and/ } \\
\text { or data management }\end{array}$ \\
\hline $\begin{array}{l}\text { Moreno Diéguez, } \\
\text { Antonio }\end{array}$ & $\begin{array}{l}\text { Complejo Hospitalario Universitario de Ferrol } \\
\text { (CHUF), Ferrol, A Coruña, Spain }\end{array}$ & Site investigator & Neuroimaging studies \\
\hline Nogueira, Víctor & Hospital Da Costa de Burela, Lugo, Spain & Site investigator /PI & $\begin{array}{c}\text { Coordination at the center } \\
\text { Evaluation of participants and/ } \\
\text { or data management }\end{array}$ \\
\hline Novo Amado, Alba & $\begin{array}{l}\text { Complejo Hospitalario Universitario de Ferrol } \\
\text { (CHUF), Ferrol, A Coruña, Spain }\end{array}$ & Site investigator & Neuroimaging studies \\
\hline Novo Ponte, Sabela & $\begin{array}{c}\text { Hospital Universitario Puerta de Hierro, Madrid, } \\
\text { Spain. }\end{array}$ & Site investigator & $\begin{array}{l}\text { Evaluation of participants and/ } \\
\text { or data management }\end{array}$ \\
\hline Ordás, Carlos & $\begin{array}{c}\text { Hospital Rey Juan Carlos, Madrid, Spain, Madrid, } \\
\text { Spain. }\end{array}$ & Site investigator & $\begin{array}{l}\text { Evaluation of participants and/ } \\
\text { or data management }\end{array}$ \\
\hline Pagonabarraga, Javier & Hospital de Sant Pau, Barcelona, Spain & Site investigator & $\begin{array}{l}\text { Evaluation of participants and/ } \\
\text { or data management }\end{array}$ \\
\hline Isabel Pareés & Hospital Ruber Internacional, Madrid, Spain & Site investigator & $\begin{array}{l}\text { Evaluation of participants and/ } \\
\text { or data management }\end{array}$ \\
\hline Pascual-Sedano, Berta & Hospital de Sant Pau, Barcelona, Spain & Site investigator & $\begin{array}{c}\text { Evaluation of participants and/ } \\
\text { or data management }\end{array}$ \\
\hline Pastor, Pau & $\begin{array}{c}\text { Hospital Universitari Mutade Terrassa, Terrassa, } \\
\text { Barcelona, Spain }\end{array}$ & Site investigator & $\begin{array}{l}\text { Evaluation of participants and/ } \\
\text { or data management }\end{array}$ \\
\hline Pérez Fuertes, Aída & $\begin{array}{l}\text { Complejo Hospitalario Universitario de Ferrol } \\
\text { (CHUF), Ferrol, A Coruña, Spain }\end{array}$ & Site investigator & Blood analysis \\
\hline Pérez Noguera, Rafael & $\begin{array}{c}\text { Hospital Universitario Virgen Macarena, Sevilla, } \\
\text { Spain }\end{array}$ & Site investigator & $\begin{array}{l}\text { Evaluation of participants and/ } \\
\text { or data management }\end{array}$ \\
\hline Planas-Ballvé, Ana & $\begin{array}{l}\text { Consorci Sanitari Integral, Hospital Moisés } \\
\text { Broggi, Sant Joan Despí, Barcelona, Spain }\end{array}$ & Site investigator & $\begin{array}{c}\text { Evaluation of participants and/ } \\
\text { or data management }\end{array}$ \\
\hline Planellas, Lluís & Hospital Clínic de Barcelona, Barcelona, Spain & Site investigator & $\begin{array}{c}\text { Evaluation of participants and/ } \\
\text { or data management }\end{array}$ \\
\hline Prats, Marian Ángeles & $\begin{array}{l}\text { Institut d'Assistència Sanitària (IAS) - Instituí } \\
\text { Cátala de la Salud. Girona, Spain }\end{array}$ & Site investigator & $\begin{array}{l}\text { Evaluation of participants and/ } \\
\text { or data management }\end{array}$ \\
\hline
\end{tabular}


Table 5: Continued.

\begin{tabular}{|c|c|c|c|}
\hline $\begin{array}{l}\text { Name (last name, first } \\
\text { name) }\end{array}$ & Location & Role & Contribution \\
\hline $\begin{array}{l}\text { Prieto Jurczynska, } \\
\text { Cristina }\end{array}$ & $\begin{array}{l}\text { Hospital Rey Juan Carlos, Madrid, Spain, Madrid, } \\
\text { Spain }\end{array}$ & Site investigator /PI & $\begin{array}{l}\text { Coordination at the center } \\
\text { Evaluation of participants and/ } \\
\text { or data management } \\
\text { Coordination at the center }\end{array}$ \\
\hline Puente, Víctor & Hospital del Mar, Barcelona, Spain & Site investigator /PI & $\begin{array}{l}\text { Coordination at the center } \\
\text { Evaluation of participants and/ } \\
\text { or data management }\end{array}$ \\
\hline $\begin{array}{l}\text { Pueyo Morlans, } \\
\text { Mercedes }\end{array}$ & $\begin{array}{l}\text { Hospital Universitario de Canarias, San Cristóbal } \\
\text { de la Laguna, Santa Cruz de Tenerife, Spain }\end{array}$ & Site investigator & $\begin{array}{c}\text { Evaluation of participants and/ } \\
\text { or data management }\end{array}$ \\
\hline Redondo, Nuria & Hospital La Princesa, Madrid, Spain & Site Investigator & $\begin{array}{c}\text { Evaluation of participants and/ } \\
\text { or data management }\end{array}$ \\
\hline $\begin{array}{l}\text { Rodríguez Méndez, } \\
\text { Luisa }\end{array}$ & $\begin{array}{l}\text { Complejo Hospitalario Universitario de Ferrol } \\
\text { (CHUF), Ferrol, A Coruña, Spain }\end{array}$ & Site investigator & Blood analysis \\
\hline $\begin{array}{l}\text { Rodríguez Pérez, } \\
\text { Amparo Belén }\end{array}$ & $\begin{array}{c}\text { Hospital General Universitario de Elche, Elche, } \\
\text { Spain }\end{array}$ & Site investigator & $\begin{array}{c}\text { Evaluation of participants and/ } \\
\text { or data management }\end{array}$ \\
\hline Roldán, Florinda & $\begin{array}{c}\text { Hospital Universitario Virgen del Rocío, Sevilla, } \\
\text { Spain }\end{array}$ & Site investigator & Neuroimaging studies \\
\hline Ruíz de Arcos, María & $\begin{array}{c}\text { Hospital Universitario Virgen Macarena, Sevilla, } \\
\text { Spain. }\end{array}$ & Site investigator & $\begin{array}{c}\text { Evaluation of participants and/ } \\
\text { or data management }\end{array}$ \\
\hline Ruíz Martínez, Javier & $\begin{array}{c}\text { Hospital Universitario Donostia, San Sebastián, } \\
\text { Spain }\end{array}$ & Site investigator & $\begin{array}{c}\text { Evaluation of participants and/ } \\
\text { or data management }\end{array}$ \\
\hline Sánchez Alonso, Pilar & $\begin{array}{l}\text { Hospital Universitario Puerta de Hierro, Madrid, } \\
\text { Spain }\end{array}$ & Site investigator & $\begin{array}{l}\text { Evaluation of participants and/ } \\
\text { or data management }\end{array}$ \\
\hline $\begin{array}{l}\text { Sánchez-Carpintero, } \\
\text { Macarena }\end{array}$ & $\begin{array}{l}\text { Complejo Hospitalario Universitario de Ferrol } \\
\text { (CHUF), Ferrol, A Coruña, Spain }\end{array}$ & Site investigator & Neuroimaging studies \\
\hline Sánchez Díez, Gema & $\begin{array}{c}\text { Hospital Universitario Ramón y Cajal, Madrid, } \\
\text { Spain }\end{array}$ & Site investigator & $\begin{array}{c}\text { Evaluation of participants and/ } \\
\text { or data management }\end{array}$ \\
\hline $\begin{array}{l}\text { Sánchez Rodríguez, } \\
\text { Antonio }\end{array}$ & $\begin{array}{c}\text { Hospital Universitario Marqués de Valdecilla, } \\
\text { Santander, Spain }\end{array}$ & Site investigator & $\begin{array}{c}\text { Evaluation of participants and/ } \\
\text { or data management }\end{array}$ \\
\hline Santacruz, Pilar & Hospital Clínic de Barcelona, Barcelona, Spain & Site investigator & $\begin{array}{c}\text { Evaluation of participants and/ } \\
\text { or data management }\end{array}$ \\
\hline Santos García, Diego & $\begin{array}{c}\text { CHUAC, Complejo Hospitalario Universitario de } \\
\text { A Coruña }\end{array}$ & Coordinator of the Project & $\begin{array}{l}\text { Coordination of the } \\
\text { COPPADIS-2015 }\end{array}$ \\
\hline $\begin{array}{l}\text { Segundo Rodríguez, } \\
\text { José Clemente }\end{array}$ & Complejo Hospitalario de Toledo, Toledo, Spain & Site investigator & $\begin{array}{c}\text { Evaluation of participants and/ } \\
\text { or data management }\end{array}$ \\
\hline Seijo, Manuel & $\begin{array}{l}\text { Complejo Hospitalario Universitario de } \\
\text { Pontevedra (CHOP), Pontevedra, Spain }\end{array}$ & Site investigator /PI & $\begin{array}{c}\text { Coordination at the center } \\
\text { Evaluation of participants and/ } \\
\text { or data management }\end{array}$ \\
\hline Sierra, María & $\begin{array}{c}\text { Hospital Universitario Marqués de Valdecilla, } \\
\text { Santander, Spain }\end{array}$ & Site investigator & $\begin{array}{l}\text { Evaluation of participants and/ } \\
\text { or data management }\end{array}$ \\
\hline Solano, Berta & $\begin{array}{l}\text { Institut d'Assistència Sanitària (IAS) - Instituí } \\
\text { Cátala de la Salud. Girona, Spain }\end{array}$ & Site investigator /PI & $\begin{array}{c}\text { Coordination at the center } \\
\text { Evaluation of participants and/ } \\
\text { or data management }\end{array}$ \\
\hline Suárez Castro, Ester & $\begin{array}{l}\text { Complejo Hospitalario Universitario de Ferrol } \\
\text { (CHUF), Ferrol, A Coruña, Spain }\end{array}$ & Site investigator & $\begin{array}{c}\text { Evaluation of participants and/ } \\
\text { or data management }\end{array}$ \\
\hline Tartari, Juan Pablo & $\begin{array}{l}\text { Hospital Universitari Mutua de Terrassa, Terrassa, } \\
\text { Barcelona, Spain }\end{array}$ & Site investigator & $\begin{array}{l}\text { Evaluation of participants and/ } \\
\text { or data management }\end{array}$ \\
\hline Valero, Caridad & Hospital Arnau de Vilanova, Valencia, Spain & Site investigator & $\begin{array}{c}\text { Evaluation of participants and/ } \\
\text { or data management }\end{array}$ \\
\hline Vargas, Laura & $\begin{array}{l}\text { Hospital Universitario Virgen del Rocío, Sevilla, } \\
\text { Spain }\end{array}$ & Site investigator & $\begin{array}{c}\text { Evaluation of participants and/ } \\
\text { or data management } \\
\text { Coordination at the center }\end{array}$ \\
\hline Vela, Lydia & Fundación Hospital de Alcorcón, Madrid, Spain & Site investigator /PI & $\begin{array}{c}\text { Evaluation of participants and/ } \\
\text { or data management }\end{array}$ \\
\hline Villanueva, Clara & $\begin{array}{c}\text { Hospital Universitario Clínico San Carlos, } \\
\text { Madrid, Spain }\end{array}$ & Site investigator & $\begin{array}{c}\text { Evaluation of participants and/ } \\
\text { or data management }\end{array}$ \\
\hline Vives, Bárbara & $\begin{array}{c}\text { Hospital Universitario Son Espases, Palma de } \\
\text { Mallorca, Spain }\end{array}$ & Site investigator & $\begin{array}{c}\text { Evaluation of participants and/ } \\
\text { or data management }\end{array}$ \\
\hline Villar, Maria Dolores & $\begin{array}{l}\text { Hospital Universitario de Canarias, San Cristóbal } \\
\text { de la Laguna, Santa Cruz de Tenerife, Spain }\end{array}$ & Site investigator & $\begin{array}{l}\text { Evaluation of participants and/ } \\
\text { or data management }\end{array}$ \\
\hline
\end{tabular}


neuropathological data are now emerging supporting the concept of the nonmotor dominant endophenotype [21], and it seems necessary in daily practice to know the frequency and the severity of NMS in PD patients, even in early PD patients, because NMS burden could be significant, and this one impacts on their QoL and contributes to disability $[7,9,15,22]$. Very recently, two PD subtypes have been suggested $[23,24]$, and it would be of great interest to know if very early PD patients with very severe NMS burden could correspond with the body-first (bottom-up) type and those with mild NMS burden with the brain-first (top-down) type.

The application of the HY.NMSB scale could have different uses: (1) a fast and relatively simple way of knowing the motor and nonmotor states of a PD patient, stratifying him/ her into a group (diagnosis value; first visit); (2) to monitor the long-term evolution of the patient (prognosis value; follow-up visits); (3) to monitor the response of a patient to a specific therapeutic intervention. In fact, the NMSS total score has been considered as the primary efficacy variable in recent trials [25], and it is known that some NMS can be improved, with dopaminergic medication or nondopaminergic medication [26]. In this context, the HY.NMSB could be used for defining a specific population or as an outcome parameter in clinical trials. For example, nabilone has very recently demonstrated to improve NMS in PD patients in a phase 2 trial [27], being an interesting possibility to identify what patients changed from a superior stage of the HY.NMSB to an inferior stage (e.g., from $2 \mathrm{C}$ to $2 \mathrm{~B}$ ). Finally, the HY-NMSB scale could be useful to indirectly estimate the patient's perception of QoL and disability. The correlation of H\&Y, NMSS, and NMS burden with QoL and disability has been frequently reported $[7-9,22]$, including in PD patients from the COPPADIS cohort $[15,18]$, but this is the first time that the relationship considering both motor stage $(\mathrm{H} \& \mathrm{Y})$ and NMS burden (NMSS) at the same time has been analyzed, and it is important because the influence of NMS burden on QoL perception is critical. An inherent limitation of the proposed classification (HY.NMSB) is the fact that the classification according to NMS is carried out taking into account the total NMS burden but without considering what exactly these symptoms are. Importantly, some NMS could help clinical practitioners to identify patients who are at different stages of the disease, such as hallucinations, fainting, inability to control body sphincters, or believing in unlikely facts [28]. Moreover, and compared with the International Parkinson and Movement Disorder Society-Nonmotor Rating Scale (MDS-NMS) [29], the NMSS collects the patient's perception about different NMS in the previous 4 weeks but does not about nonmotor fluctuations.

A very important limitation is that our sample is not fully representative of the $\mathrm{PD}$ population due to inclusion and exclusion criteria (i.e., age limit, no dementia, no severe comorbidities, and no second line therapies) which subsequently entails a bias toward early PD. The majority of the patients from this cohort were in the stage I or II of the H\&Y (90.4\%), so the same analysis with the proposed classification should be carried out in a cohort with more patients in advanced stages of H\&Y. In spite of this and importantly, during the first 5 to 10 years of the disease, many patients with PD will be in stage II of the H\&Y, and introducing the NMS burden will help to differentiate the degree of nonmotor affectation, that importantly correlates with QoL perception. In other words, the results of the present study are applicable for a long time to the majority of PD patients, especially in early young PD patients. Second, all scales or questionnaires used for assessing motor and NMS are validated except PQ-10 [8, 15]. Third, NMS were recorded with the NMSS, but specifically, as we commented nonmotor fluctuations were not identified [30]. Fourth, the OFF state (12 hours without taking medication) was considered for defining the H\&Y stage because it represents a more natural state of the disease less conditioned by the symptomatic effect of the medication. Moreover, in PD patients with motor fluctuations, the symptoms during the OFF state mostly impact on QoL and autonomy. In any case, previously, similar results applying the HY.NMSB were observed when the H\&Y stage was defined during the ON state in 149 PD patients from the CASINO cohort [8, 31]. In the COPPADIS cohort, the results were similar as well when the $\mathrm{H} \& \mathrm{Y}$ was defined during the ON state in those PD patients with motor fluctuations (data not shown). Fifth, the time it took to administer the HY.BMSB scale was not calculated. Finally, this is a cross-sectional study, but the aim of the COPPADIS-2015 study [12] is to follow-up the cohort for 5 years, so changes in HY.NMSB and the relationship with changes in other variables could be analyzed.

In conclusion, this is the first time that a specific scale combing the H\&Y stage and the NMSS (HY.NMSB scale) is applied in PD patients for knowing the relationship with the patient's QoL perception and disability regarding the stage. The HY.NMSB scale is simple and reflects the degree of patient involvement more accurately than the H\&Y. Patients with a lower H\&Y stage may be more affected if they have a greater NMS burden. These results need to be replicated in a larger and well-distributed cohort of patients by motor stage.

\section{Appendix}

\section{A. COPPADIS Study Group}

The authors in the COPPADIS Study Group have been listed in Table 5.

\section{Abbreviations}

ADLS: $\quad$ Schwab and England Activities of Daily

BDI: $\quad$ Living Scale

EUROHIS- European Health Interview Survey-Quality

QOL8: of Life 8 Item-Index

FOGQ: $\quad$ Freezing of gait questionnaire

H\&Y: $\quad$ Hoenh and Yahr

NMS: $\quad$ Nonmotor symptoms

NMSB: $\quad$ Nonmotor symptoms burden

NMSS: $\quad$ Nonmotor symptoms scale

NPI: $\quad$ Neuropsychiatric inventory

PD: $\quad$ Parkinson's disease

PD-CRS: $\quad$ Parkinson's Disease Cognitive Rating Scale 
PDQ-39SI: $\quad$ 39-Item Parkinson's Disease Quality of Life Questionnaire Summary Index

PDSS: $\quad$ Parkinson's Disease Sleep Scale

QoL: Quality of life

QUIP-RS: Questionnaire for Impulsive-Compulsive Disorders in Parkinson's Disease-Rating Scale

UPDRS: Unified Parkinson's Disease Rating Scale

VAFS: Visual Analog Fatigue Scale

VAS pain: Visual Analog Scale Pain.

\section{Data Availability}

The protocol and the statistical analysis plan are available on request. Deidentified participants data are not available for legal and ethical reasons.

\section{Conflicts of Interest}

Santos García D has received honoraria for educational presentations and advice service by AbbVie, UCB Pharma, Lundbeck, KRKA, Zambon, Bial, Italfarmaco, and Teva. Paz González JM has received honoraria for educational presentations and/or advice service by UCB Pharma, Lundbeck, KRKA, and Zambon. Jesús $S$ has received honoraria from AbbVie, Bial, Merz, UCB, and Zambon and holds the competitive contract "Juan Rodés" supported by the Instituto de Salud Carlos III. She has received grants from the Spanish Ministry of Economy and Competitiveness (PI18/ 01898) and the Consejería de Salud de la Junta de Andalucía (PI-0459-2018). Aguilar M obtained from UCB and Schwabe with assistance to a Congress and Nutricia with assistance to a Congress and payment of lecture. Planellas LL has received travel bursaries grant from AbbVie. García Caldentey J has received honoraria for educational presentations and advice service by Qualigen, Nutricia, AbbVie, Italfarmaco, UCB Pharma, Lundbeck, Zambon, Bial and Teva. Caballol $\mathrm{N}$ has received honoraria from Bial, Italfarmaco, Qualigen, Zambon, UCB, Teva, and KRKA and sponsorship from Zambon, TEVA, and AbbVie for attending medical conferences. Legarda I has received honoraria for educational presentations and advice service by AbbVie, UCB Pharma, Zambon, Bial, and Teva. Hernández Vara $\mathrm{J}$ has received travel bursaries and educational grants from AbbVie and has received honoraria for educational presentations from AbbVie, Teva, Bial, Zambon, Italfarmaco, and SanofiGenzyme. Cabo I has received honoraria for educational presentations and advice service by AbbVie, Zambon, and Bial. López Manzanares L compensated advisory services, consulting, research grant support, or speaker honoraria from AbbVie, Acorda, Bial, Intec Pharma, Italfarmaco, Pfizer, Roche, Teva, UCB, and Zambon. Ávila Rivera MA has received honoraria from Zambon, UCB Pharma, Qualigen, Bial, and Teva and ana sponsorship from Zambon and Teva for attending conferences.

Puente $\mathrm{V}$ has served as consultant for AbbVie and Zambon and has received grant/research from AbbVie. García Moreno JM has received honoraria for educational presentations and advice service by AbbVie, Ital-Pharma,
Lundbeck, Merz, KRKA, UCB, Pharma, Zambon, Bial, and Teva. Solano Vila B has received honoraria for educational presentations and advice service by UCB, Zambon, Teva, AbbVie, Bial. Álvarez Sauco M has received honoraria for educational presentations and advice service by AbbVie, UCB Pharma, Zambon, Bial, and Teva. Vela L has received honoraria for educational presentations and advice service by AbbVie, UCB Pharma, Lundbeck, KRKA, Zambon, Bial, and Teva. Escalante $S$ has received honoraria for educational presentations and advice service by AbbVie, Zambon, and Bial. Cubo $\mathrm{E}$ has travel grants from AbbVie, Allergan, and Boston; lecturing honoraria from AbbVie and International Parkinson's Disease Movement Disorder Society. Carrillo Padilla F has received honoraria from Zambon (SEN Congress assistance). Martínez Castrillo JC has received research support from Lundbeck, Italfarmaco, Allergan, Zambon, Merz, and AbbVie and speaking honoraria from AbbVie, Bial, Italfarmaco, Lundbeck, Krka, TEVA, UCB, Zambon, Allergan, Ipsen, and Merz. Sánchez Alonso $\mathrm{P}$ has received honoraria for educational presentations and advice service by AbbVie, UCB Pharma, Lundbeck, KRKA, Zambon, Bial, and Teva. Alonso Losada MG has received honoraria for educational presentations and advice service by Zambon and Bial. López Ariztegui N has received honoraria for educational presentations and advice service by AbbVie, Italfarmaco, Zambon, and Bial. Gastón I has received research support from AbbVie and Zambon and has served as a consultant for AbbVie, Exelts, and Zambon. Kulisevsky J has obtained the following: (1) consulting fees: Roche and Zambon; (2) honoraria (e.g., lecture fees): Zambon, Teva, Bial, and UCB; (3) research funding: Roche, Zambon, Ciberned, Instituto de SaludCarlos III, and Fundació La Marató De TV3. Blázquez Estrada $M$ has received honoraria for educational presentations and advice service by AbbVie, Abbott, UCB Pharma, Allergan, Zambon, Bial, and Qualigen. Seijo M has received honoraria for educational services from KRKA, UCB, Zambon, and Bial and travel grants from Daiichi and Roche. Ruiz Martínez J has received honoraria for educational presentations, attending medical conferences, and advice service by AbbVie, UCB Pharma, Zambon, Italfarmaco, Bial, and Teva. Valero $\mathrm{C}$ has received honoraria for educational services from Zambon AbbVie and UCB. Kurtis $M$ has received honoraria from Bial, the Spanish Neurology Society, and the International and Movement Disorders Society. de Fábregues $\mathrm{O}$ has received honoraria for educational presentations and advice service by Bial, Zambon, AbbVie, KRKA, and Teva. González Ardura J has received honoraria for speaking from Italfarmaco, Krka, Genzyme, UCB, Esteve, Psyma iberica marketing research SL and Ferrer, course grant from Teva, and travel grant from Merck. López Díaz L has received honoraria from UCB, Lundbeck, and KRKA. Mir P has received honoraria from AbbVie, Abbott, Allergan, Bial, Merz, UCB, and Zambon and has received grants from the Spanish Ministry of Economy and Competitiveness (PI16/01575) cofounded by ISCIII (Subdirección General de Evaluación y Fomento de la Investigación) and by Fondo Europeo de Desarrollo Regional (FEDER), the Consejería de Economía, 
Innovación, Ciencia y Empleo de la Junta de Andalucía (CVI-02526, CTS-7685), the Consejería de Salud y Bienestar Social de la Junta de Andalucía (PI-0437-2012, PI0471-2013), the Sociedad Andaluza de Neurología, the Jacques and Gloria Gossweiler Foundation, the Fundación Alicia Koplowitz, and the Fundación Mutua Madrileña. Martínez-Martin P has received honoraria from Editorial Viguera for lecturing in courses, International Parkinson and Movement Disorder Society (IPMDS) for management of the Program on Rating Scales, Air Liquide, AbbVie, and HM Hospitales de Madrid for advice in clinic-epidemiological studies; license fee payments for the King's Parkinson's Disease Pain scale; financial support by the IPMDS for attending the IPMDS International Congress 2018; and grant for research from IPMDS for development and validation of the MDS-NMS. Borrué $C$, Ordás C, Catalán MJ, Nogueira V, González Aramburu I, Cosgaya M, Pastor P, De Deus T, Cores Bartolomé C, Valdés Aymerich L, Muñoz Enríquez JG, and Suárez declare that they have no conflicts of interest.

\section{Authors' Contributions}

Santos García D was responsible for conception, organization, and execution of the project; statistical analysis; writing of the first draft of the manuscript; and recruitment and/or evaluation of participants. De Deus T Suárez E, Jesús S Aguilar M, Pastor P, Planellas LL, Cosgaya M, García Caldentey J, Caballol N, Legarda I, Hernández Vara J, Cabo I, López Manzanares L, González Aramburu I, Ávila Rivera MA, Catalán MJ, Nogueira V, Puente V, García Moreno JM, Borrué C, Solano Vila B, Álvarez Sauco M, Vela L, Escalante S, Cubo E, Carrillo Padilla F, Martínez Castrillo JC, Sánchez Alonso P, Alonso Losada MG, López Ariztegui N, Gastón I, Kulisevsky J, Blázquez Estrada M, Seijo M, Ruiz Martínez J, Valero C, Kurtis M, de Fábregues O, González Ardura J, Ordás C, López Díaz L, and Mir P contributed to review and critique and recruitment and/or evaluation of participants. Paz González JM, Cores Bartolomé C, Valdés Aymerich L, and Muñoz Enríquez JG managed review and critique. Martínez-Martin P did review and critique and supervision.

\section{Acknowledgments}

The authors would like to thank all patients and their caregivers who collaborated in this study. Many thanks also are due to Fundación Curemos el Parkinson (http://www. curemoselparkinson.org) and Alpha Bioresearch (http:// www.alphabioresearch.com).

\section{Supplementary Materials}

Table 1 SM. Disease-related characteristics, motor and nonmotor symptoms, and autonomy for activities of daily living and quality of life in PD patients $(n=603)$. Table 2 SM. Multiple regression model for PDQ-39SI as dependent variable. (Supplementary Materials)

\section{References}

[1] C. W. Olanow and J. A. Obeso, "The significance of defining preclinical or prodromal Parkinson's disease," Movement Disorders, vol. 27, no. 5, pp. 666-669, 2012.

[2] M. M. Hoehn and M. D. Yahr, "Parkinsonism: onset, progression, and mortality," Neurology, vol. 17, no. 5, p. 427, 1967.

[3] C. G. Goetz, W. Poewe, and O. Rascol, "Movement disorder society task force report on the hoehn and yahr staging scale: status and recommend the authorsions the movement disorder society task force on rating scales for Parkinson's disease," Movement Disorders, vol. 19, no. 9, pp. 1020-1028, 2004.

[4] S. Fanhn, R. L. Elton, and Members of the UPDRS Development Committee, "Unified Parkinson's disease rating scale," in Recent developments in Parkinson's disease, S. Fahn, C. D. Marsden, D. B. Calne, and M. Goldstein, Eds., pp. 153-64, Macmillan Health Care Information, Florham Park, NJ, USA, 1987.

[5] C. G. Goetz, B. C. Tilley, S. R. Shaftman et al., "Movements disorder society-sponsored revision of the unisied partinson's disease rating scale (mds-updrs): scale presentation and clinimetric testing results," Movement Disorders, vol. 23, no. 15, pp. 2129-2170, 2008.

[6] K. R. Chaudhuri, P. Martinez-Martin, and A. H. V. Schapira, "International multicenter pilot study of the first comprehensive self-completed nonmotor symptoms questionnaire for Parkinson's disease: the NMSQuest studyInternational multicenter pilot study of the first," Movement Disorders, vol. 21, no. 7, pp. 916-923, 2006.

[7] K. R. Chaudhuri, P. Martinez-Martin, and R. G. Brown, “The metric properties of a novel non-motor symptoms scale for Parkinson's disease: results from an international pilot studyThe metric properties of a novel non-motor symptoms scale for Parkinson's disease: Results from an international pilot study," Movement Disorders, vol. 22, no. 13, pp. 1901-1911, 2007.

[8] D. Santos-García and R. de la Fuente-Fernández, "Impact of non-motor symptoms on health-related and perceived quality of life in Parkinson's disease," Journal of the Neurological Sciences, vol. 332, no. 1-2, pp. 136-140, 2013.

[9] P. Martinez-Martin, C. Rodriguez-Blazquez, M. M. Kurtis, and K. R. Chaudhuri, "The impact of non-motor symptoms on health-related quality of life of patients with Parkinson's disease," Movement Disorders, vol. 26, no. 3, pp. 399-406, 2011.

[10] P. Martinez-Martin and K. Ray Chaudhuri, "prehensive grading of Parkinson's disease using motor and non-motor assessments: addressing a key unmet need," Expert Review of Neurotherapeutics, vol. 18, no. 1, pp. 41-50, 2018.

[11] K. Ray Chaudhuri, J. M. Rojo, A. H. Schapira et al., "A proposal for a comprehensive grading of Parkinson's disease severity combining motor and non-motor assessments: meeting an unmet need," PLoS One, vol. 8, Article ID e57221, 2013.

[12] D. Santos-García, P. Mir, E. Cubo et al., "COPPADIS-2015 (Cohort of Patients with Parkinson's disease in Spain, 2015), a global--clinical evaluations, serum biomarkers, genetic studies and neuroimaging--prospective, multicenter, noninterventional, long-term study on Parkinson's disease progression," BMC Neurology, vol. 16, p. 26, 2016.

[13] D. Santos García, S. Jesús, M. Aguilar et al., "COPPADIS-2015 (COhort of Patient's with Parkinson's disease in Spain, 2015): 
An ongoing global Parkinson's d.isease Pr.oject about d.isease progression with more than 1,000 subjects included. results from the baseline evaluation," European Journal of Neurology, vol. 26, pp. 1399-407, 2019.

[14] C. Jenkinson, R. Fitzpatrick, V. Peto, R. Greenhall, and N. Hyman, "The Parkinson's disease questionnaire (PDQ3.9): development and validation of a Parkinson's disease summary index score," Age and Ageing, vol. 26, no. 5, pp. 353-357, 1997.

[15] D. Santos García, T. de Deus Fonticoba, and E. Suárez Castro, "Non-motor symptoms burden, mood, and gait problems are the most significant factors contributing to a poor quality of life in non-demented Parkin.son's dis.ease patien.ts: Results from the COPPADIS Study Cohort," Parkinsonism \& Related Disorders, vol. 66, pp. 151-157, 2019.

[16] N. S. d. Rocha, M. J. Power, D. M. Bushnell, and M. P. Fleck, "The EUROHIS-QOL 8-item index: comparative psychometric properties to its parent WHOQOL-BREF," Value in Health, vol. 15, no. 3, pp. 449-457, 2012.

[17] R. S. Schwab and A. C. England, Parkinson's disease, pp. 152-7, E. And S. Livingstone, Edinburgh, Scotland, 1969.

[18] D. Santos-García, T. de Deus-Fonticoba, E. Suárez Castro et al., "The impact of freezing of. gait on function.al dependency in Parkinson's disease with regard to motor phenotype," Neurological Sciences, vol. 41, 2020.

[19] D. J. Burn, S. Landau, J. V. Hindle, M. Samuel, K. C. Wilson, and C. S. Hurt, "Parkinson's disease motor subtypes and mood," Movement Disorders, vol. 27, no. 3, pp. 379-386, 2012.

[20] A. Todorova, P. Jenner, and K. Ray Chaudhuri, "Non-motor Parkinson's: integral to motor Parkinson's, yet often neglected," Practical Neurology, vol. 14, no. 5, pp. 310-322, 2014.

[21] A. Sauerbier, P. Jenner, and A. Todorova, "Non motor subtypes and Parkinson's disease," Parkinsonism \& Related Disorders, vol. 22, no. 1, pp. S41-S46, 2016.

[22] K. M. Prakash, N. V. Nadkarni, W.-K. Lye, M.-H. Yong, and E.-K. Tan, "The impact of non-motor symptoms on the quality of life of Parkinson's disease patients: a longitudinal study," European Journal of Neurology, vol. 23, no. 5, p. 854, 2016.

[23] M. L. Arotcarena, S. Dovero, A. Prigent et al., "Bidirectional gut-to-brain and brain-to-gut propagation of synucleinopathy in non-human primates," Brain, vol. 143, 2020.

[24] J. Horsager, K. B. Andersen, K. Knudsen et al., "Brain-first versus body-first Parkinson's disease: a multimodal imaging case-control study," Brain, vol. 143, 2020.

[25] A. Antonini, L. Bauer, and E. Dohin, "Effects of rotigotine transdermal patch in patients with Parkinson's disease presenting with non-motor symptoms - results of a double-blind, randomized, placebo-controlled trial," European Journal of Neurology, vol. 22, no. 10, pp. 1400-1407, 2015.

[26] K. Seppi, K. Ray Chaudhuri, and M. Coelho, "Update on treatments for nonmotor symptoms of Parkinson's disease-an evidence-based medicine review," Movement Disorders, vol. 34, no. 2, pp. 180-198, 2019.

[27] M. Peball, F. Krismer, H. G. Knaus et al., "Non-Motor Symptoms in Parkinson's Disease are Reduced by Nabilone," Annals of Neurology, vol. 88, no. 4, pp. 712-722, 2020.

[28] R. Armañanzas, C. Bielza, K. R. Chaudhuri, P. MartinezMartin, and P. Larrañaga, "Unveiling relevant non- Parkinson's disease severity symptoms using a machine learning approach," Artificial Intelligence in Medicine, vol. 58, no. 3, pp. 195-202, 2013.

[29] K. R. Chaudhuri, A. Schrag, D. Weintraub, and A. Rizos, "The movement disorder society nonmotor rating scale: Initial validation study," Movement Disorders, vol. 35, no. 1, pp. 116-133, 2020.

[30] R. Martínez-Fernández, E. Schmitt, P. Martinez-Martin, and P. Krack, "The hidden sister of motor flucsuations in Parkinson's disease: A review on nonmotor fluctuations," Movement Disorders, vol. 31, no. 8, pp. 1080-94, 2016.

[31] I. Expósito-Ruiz, E. Suárez-Castro, D. Santos-García et al., "Relación entre calidad de vida, Hoehn\&Yahr y síntomas no motores: el porqué de usar una escala que combine el estadio motor y la afectación no motora en pacientes con enfermedad de Parkinson. Oral Communication," in Proceedings of the Annual Meeting of the Spanish Society of Neurology, Santiago De Compostela, Spain, September 2015. 